\title{
The Mabuchi Completion of the Space of Kähler Potentials
}

\author{
Tamás Darvas*
}

To Andrea.

\begin{abstract}
Suppose $(X, \omega)$ is a compact Kähler manifold. Following Mabuchi, the space of smooth Kähler potentials $\mathcal{H}$ can be endowed with a Riemannian structure, which induces an infinite dimensional path length metric space $(\mathcal{H}, d)$. We prove that the metric completion of $(\mathcal{H}, d)$ can be identified with $\left(\mathcal{E}^{2}(X, \omega), \tilde{d}\right)$, and this latter space is a complete non-positively curved geodesic metric space. In obtaining this result, we will rely on envelope techniques which allow for a treatment in a very general context. Profiting from this, we will characterize the pairs of potentials in $\operatorname{PSH}(X, \omega)$ that can be connected by weak geodesics and we will also give a characterization of $\mathcal{E}(X, \omega)$ in this context.
\end{abstract}

\section{Introduction}

Given $\left(X^{n}, \omega\right)$, a connected compact Kähler manifold, the space of smooth Kähler potentials is the set

$$
\mathcal{H}=\left\{u \in C^{\infty}(X) \mid \omega+i \partial \bar{\partial} u>0\right\} .
$$

Using the $\partial \bar{\partial}$-lemma of Hodge theory, this set can be identified (up to a constant) with the space of Kähler metrics that are cohomologous to $\omega$. $\mathcal{H}$ is a Fréchet manifold, and as such one can endow it with different Riemannian structures that induce path length metric spaces on $\mathcal{H}$. One such Riemannian structure was introduced by E. Calabi [Ca] in 1954 (see also [Cl]), who proposed to find the metric completion of $\mathcal{H}$ using his metric. This was eventually carried out by B. Clarke and Y. Rubinstein in [CR]. As pointed out in [CR], it would be desirable, although much harder, to find the metric completion of $\mathcal{H}$ with respect to the much more studied Mabuchi metric. The main theorem of this paper answers this question completely, confirming a conjecture of V. Guedj $G$ ] in the process.

\subsection{Background}

To state our main result it is necessary to recall some facts about the Mabuchi geometry of $\mathcal{H}$. For a more exhaustive survey we refer to Bl1]. For $v \in \mathcal{H}$ one can identify $T_{v} \mathcal{H}$ with $C^{\infty}(X)$. After Mabuchi [M], we introduce the following Riemannian metric on $\mathcal{H}$ :

$$
\langle\xi, \eta\rangle_{v}:=\int_{X} \xi \eta(\omega+i \partial \bar{\partial} v)^{n}, \quad \xi, \eta \in T_{v} \mathcal{H} .
$$

*Research supported by NSF grant DMS1162070. 2010 Mathematics subject classification 53C55, 32W20, 32U05. 
As discovered independently by Semmes [Se] and Donaldson [Do], the geodesic equation of this metric can be written as a complex Monge-Ampère equation. Let $(0,1) \ni$ $t \rightarrow u_{t} \in \mathcal{H}$ be a smooth curve, $S=\{s \in \mathbb{C}: 0<\operatorname{Re} s<1\}$ and let $\tilde{\omega}$ be the pullback of the Kähler form $\omega$ to the product $S \times X$. Let $u \in C^{\infty}(S \times X)$ be the complexification of $t \rightarrow u_{t}$, defined by $u(s, x):=u_{\text {Res }}(x)$. Then $t \rightarrow u_{t}$ is a geodesic if and only if the following equation is satisfied for $u$ :

$$
\left(\tilde{\omega}+i \partial_{S \times X} \bar{\partial}_{S \times X} u\right)^{n+1}=0 .
$$

In the future we will not make a difference between a curve and its complexification.

Unfortunately, given $u_{0}, u_{1} \in \mathcal{H}$ there may be no smooth geodesic connecting them ([LV, DL, Da1]). As shown by X. X. Chen [C] (with complements by Z. Blocki [Bl2]), there exists certain weak geodesics connecting points of $\mathcal{H}$. To define these curves, one is driven into the world of pluripotential theory. The set of $\omega$-plurisubharmonic functions is a natural generalization of $\mathcal{H}$ :

$$
\operatorname{PSH}(X, \omega)=\left\{u \equiv-\infty \text { or } u \in C^{\uparrow}(X) \text { with } \omega+i \partial \bar{\partial} u \geq 0 \text { in the sense of currents }\right\}
$$

where $C^{\uparrow}(X)$ is the set of upper semi-continuous integrable functions on $X$. Chen proved that there exists a curve

$$
[0,1] \ni t \rightarrow u_{t} \in \mathcal{H}_{\Delta}=\left\{u \in \operatorname{PSH}(X, \omega) \mid \Delta u \in L^{\infty}(X)\right\}
$$

connecting $u_{0}, u_{1}$ such that the map $(t, x) \rightarrow u_{t}(x)$ has bounded Laplacian, and the complexification of $u$ (again denoted by $u$ ) satisfies (11) in the weak sense of BedfordTaylor [C]. For this curve we do not have $u_{t} \in \mathcal{H}, t \in(0,1)$, however many of its properties mimic those of actual geodesics (Section 2.1, see also [CC, CT]).

As usual, the length of a smooth curve $[0,1] \ni t \rightarrow \alpha_{t} \in \mathcal{H}$ is computed by the formula:

$$
l(\alpha)=\int_{0}^{1} \sqrt{\int_{X} \dot{\alpha}_{t}^{2}\left(\omega+i \partial \bar{\partial} \alpha_{t}\right)^{n}} d t .
$$

The distance $d\left(u_{0}, u_{1}\right)$ between $u_{0}, u_{1} \in \mathcal{H}$ is the infimum of the length of smooth curves joining $u_{0}$ and $u_{1}$. Chen proved that $d\left(u_{0}, u_{1}\right)=0$ if and only if $u_{0}=u_{1}$, thus $(\mathcal{H}, d)$ is a metric space [C].

A curve $(0,1) \ni t \rightarrow v_{t} \in \operatorname{PSH}(X, \omega)$ is called a subgeodesic segment if its complexification $v: S \times X \rightarrow \mathbb{R}$ is an $\omega$-plurisubharmonic function. If additionally $v$ is locally bounded and satisfies (11) in the sense of Bedford-Taylor, then $t \rightarrow v_{t}$ is called a weak geodesic segment.

\subsection{The Main Result}

One of the goals of this work is to generalize the notion of weak geodesic, so that given $u_{0}, u_{1} \in \operatorname{PSH}(X, \omega)$ there is a weak geodesic $(0,1) \ni t \rightarrow u_{t} \in \operatorname{PSH}(X, \omega)$ connecting $u_{0}$ with $u_{1}$, i.e. $\lim _{t \rightarrow 0,1}=u_{0,1}$ in some suitable sense explained below. As we will see, this is not always possible, but by the end of this paper we partially attain this goal (Theorem 5).

When $u_{0}, u_{1}$ are bounded, Berndtsson constructed such weak geodesic $t \rightarrow u_{t}$ (Section 2.2 and $[\mathrm{Br}])$. In the unbounded case we define $t \rightarrow u_{t}$ as a decreasing limit of 
bounded weak geodesics. Let $\left\{u_{0}^{k}\right\}_{k \in \mathbb{N}},\left\{u_{1}^{k}\right\}_{k \in \mathbb{N}} \subset \mathcal{H}$ be sequences decreasing to $u_{0}$ and $u_{1}$ respectively. By $[\mathrm{BK}]$ it is always possible to find such an approximating sequence. Let $u_{t}^{k}:[0,1] \rightarrow \mathcal{H}_{\Delta}$ be the weak geodesic joining $u_{0}^{k}, u_{1}^{k}$, whose existence was proved by Chen. We define $t \rightarrow u_{t}$ as the decreasing limit:

$$
u_{t}=\lim _{k \rightarrow+\infty} u_{t}^{k}, t \in(0,1)
$$

The curve $(0,1) \ni t \rightarrow u_{t} \in \operatorname{PSH}(X, \omega)$ we just constructed may be identically equal to $-\infty$, however one can see that it is independent of the choice of approximating sequences, hence well defined. Indeed, $u$ is the upper envelope of a family $\mathcal{S}$ of subgeodesics:

$$
u=\sup _{v \in \mathcal{S}} v
$$

where $\mathcal{S}=\left\{(0,1) \ni t \rightarrow v_{t} \in \operatorname{PSH}(X, \omega)\right.$ is a subgeodesic with $\left.\lim _{t \rightarrow 0,1} v_{t} \leq u_{0,1}\right\}$.

One would also like to extend the path length metric $d$ on $\mathcal{H}$ to $\operatorname{PSH}(X, \omega)$ the obvious way, i.e. $\tilde{d}\left(u_{0}, u_{1}\right)=\lim _{k \rightarrow \infty} d\left(u_{0}^{k}, u_{1}^{k}\right)$, however it is not clear if this limit even exists or if it is independent of the approximating sequences. As we shall see shortly, to make this precise one has to restrict the definition of $\tilde{d}$ to a subset of $\operatorname{PSH}(X, \omega)$. Before we can identify this domain, we need to recall some facts about finite energy classes on compact Kähler manifolds introduced by Guedj-Zeriahi GZ1. For $v \in \operatorname{PSH}(X, \omega)$ let $v_{h}=\max \{v,-h\}, h \in \mathbb{R}$. By an application of the comparison principle, it follows that the Borel measures $\mathbb{1}_{\{v>-h\}}\left(\omega+i \partial \bar{\partial} v_{h}\right)^{n}$ are increasing for $h>0$. By definition, $v \in \mathcal{E}(X, \omega)$ if

$$
\lim _{h \rightarrow \infty} \int_{X} \mathbb{1}_{\{v>-h\}}\left(\omega+i \partial \bar{\partial} v_{h}\right)^{n}=\int_{X} \omega^{n}=: \operatorname{Vol}(X) .
$$

Suppose $\chi: \mathbb{R} \cup\{-\infty\} \rightarrow \mathbb{R}$ is a continuous increasing function, with $\chi(0)=0$ and $\chi(-\infty)=-\infty$. Such $\chi$ is referred to as a weight. The set of all weights is denoted by $\mathcal{W}$. Given $v \in \mathcal{E}(X, \omega)$ we have $v \in \mathcal{E}_{\chi}(X, \omega)$ if

$$
E_{\chi}(v)=\lim _{h \rightarrow \infty} \int_{\{v>-h\}} \chi\left(v_{h}\right)\left(\omega+i \partial \bar{\partial} v_{h}\right)^{n}>-\infty .
$$

We will be most interested in $\mathcal{E}^{2}(X, \omega)$, this being the finite energy class given by the weight $\chi(t)=-(-t)^{2}$ (see Section 2.3). Given $u_{0}, u_{1} \in \mathcal{E}^{2}(X, \omega)$ and decreasing approximating sequences $u_{0}^{k}, u_{1}^{k} \in \mathcal{H}$, we define $\tilde{d}\left(u_{0}, u_{1}\right)$, as promised, by the formula:

$$
\tilde{d}\left(u_{0}, u_{1}\right)=\lim _{k \rightarrow \infty} d\left(u_{0}^{k}, u_{1}^{k}\right) .
$$

We will prove that this definition is independent of the choice of decreasing approximating sequences and $\tilde{d}$ is a metric on $\mathcal{E}^{2}(X, \omega)$. Moreover, for $t \rightarrow u_{t}$ as defined in (3) , we have $u_{t} \in \mathcal{E}^{2}(X, \omega), t \in(0,1)$ and the following theorem holds, which is our main result:

Theorem 1 (Theorem 6.1, Theorem 9.2). $\left(\mathcal{E}^{2}(X, \omega), \tilde{d}\right)$ is a complete non-positively curved geodesic metric space, with geodesic segments joining $u_{0}, u_{1} \in \mathcal{E}^{2}(X, \omega)$ given by (3). Furthermore, $\left(\mathcal{E}^{2}(X, \omega), \tilde{d}\right)$ can be identified with the metric completion of $(\mathcal{H}, d)$.

This result was proved for toric Kähler manifolds and conjectured to hold in general by V. Guedj in a preliminary version of $[\mathrm{G}]$. 
Let us recall that a geodesic metric space $(M, \rho)$ is a metric space for which any two points can be connected with a geodesic. By a geodesic connecting two points $a, b \in M$ we understand a curve $\alpha:[0,1] \rightarrow M$ such that $\alpha(0)=a, \alpha(1)=b$ and

$$
\rho\left(\alpha\left(t_{1}\right), \alpha\left(t_{2}\right)\right)=\left|t_{1}-t_{2}\right| \rho(a, b)
$$

for any $t_{1}, t_{2} \in[0,1]$. Furthermore, a geodesic metric space $(M, \rho)$ is non-positively curved (in the sense of Alexandrov) if for any distinct points $q, r \in M$ there exists a geodesic $\gamma:[0,1] \rightarrow M$ joining $q, r$ such that for any $s \in\{\gamma\}$ and $p \in M$ the following inequality is satisfied:

$$
\rho(p, s)^{2} \leq \lambda \rho(p, r)^{2}+(1-\lambda) \rho(p, q)^{2}-\lambda(1-\lambda) \rho(q, r)^{2},
$$

where $\lambda=\rho(q, s) / \rho(q, r)$. A basic property of non-positively curved metric spaces is that geodesic segments joining different points are unique. For more about these spaces we refer to $[\mathrm{BH}]$.

As a consequence of the last theorem, using the estimates of Berndtsson $\mathrm{Br}$, Błocki [Bl3], [BD, Corollary 4.7] (see also [H]) and Theorem 5, we obtain that $\left(\mathcal{E}^{2}(X, \omega), \tilde{d}\right.$ ) has many special totally geodesic dense subspaces, with this answering positively questions raised in $[\mathrm{H}]$ :

Corollary 2 (Corollary 6.13). The following sets are totally geodesic dense subspaces of $\left(\mathcal{E}^{2}(X, \omega), \tilde{d}\right)$ :

(i) $\mathcal{H}_{\Delta}=\left\{u \in \operatorname{PSH}(X, \omega) \mid \Delta u \in L^{\infty}(X)\right\}$;

(ii) $\mathcal{H}_{0,1}=\operatorname{PSH}(X, \omega) \cap \operatorname{Lip}(X)$;

(iii) $\mathcal{H}_{0}=\operatorname{PSH}(X, \omega) \cap L^{\infty}(X)$;

\subsection{Further Results}

The proof of Theorem 1 involves the study of certain upper envelopes. The tools we develop will allow us to treat weak geodesics in a very general context, even beyond the class $\mathcal{E}^{2}(X, \omega)$, which will be further explored in the paper [Da3]. Given $b_{0}, b_{1} \in C^{\uparrow}(X)$, one can define the envelopes

$$
\begin{gathered}
P\left(b_{0}\right)=\sup \left\{\psi \leq b_{0}: \psi \in \operatorname{PSH}(X, \omega)\right\}, \\
P\left(b_{0}, b_{1}\right)=P\left(\min \left(b_{0}, b_{1}\right)\right) .
\end{gathered}
$$

As the upper semi-continuous regularization $\operatorname{usc}\left(P\left(b_{0}\right)\right)$ is an element of $\left\{\psi \leq b_{0}: \psi \in\right.$ $\operatorname{PSH}(X, \omega)\}$ and $P\left(b_{0}\right) \leq \operatorname{usc}\left(P\left(b_{0}\right)\right)$, it follows that $P\left(b_{0}\right) \in \operatorname{PSH}(X, \omega)$ and similarly $P\left(b_{0}, b_{1}\right) \in \operatorname{PSH}(X, \omega)$. The motivation for the study of envelopes of the type $P\left(b_{0}, b_{1}\right)$ came from the following identity found in [DR] (see also Section 2.2):

$$
\inf _{t \in(0,1)} u_{t}=P\left(u_{0}, u_{1}\right)
$$

where $u_{0}, u_{1} \in \mathcal{H}_{0}=\operatorname{PSH}(X, \omega) \cap L^{\infty}$ and $(0,1) \ni t \rightarrow u_{t} \in \mathcal{H}_{0}$ is the weak geodesic connecting them. Further evidence that properties of the envelope $P\left(u_{0}, u_{1}\right)$ are tied 
together with the metric structure of the space $\mathcal{H}$ is given by the 'Pythagorean' identity of Theorem 8.1:

$$
\tilde{d}\left(u_{0}, u_{1}\right)^{2}=\tilde{d}\left(u_{0}, P\left(u_{0}, u_{1}\right)\right)^{2}+\tilde{d}\left(P\left(u_{0}, u_{1}\right), u_{1}\right)^{2} .
$$

Our very first result about these envelopes says that the classes $\mathcal{E}_{\chi}(X, \omega)$ are closed under the operation $\left(u_{0}, u_{1}\right) \rightarrow P\left(u_{0}, u_{1}\right)$.

Theorem 3 (Theorem 3.6). If $\chi \in \mathcal{W}$ and $u_{0}, u_{1} \in \mathcal{E}_{\chi}(X, \omega)$, then $P\left(u_{0}, u_{1}\right) \in \mathcal{E}_{\chi}(X, \omega)$. More precisely, if $N \in \mathbb{R}$ is such that $\chi \circ u_{0}, \chi \circ u_{1} \leq N$, then we have:

$$
E_{\chi}\left(P\left(u_{0}, u_{1}\right)\right) \geq E_{\chi}\left(u_{0}\right)+E_{\chi}\left(u_{1}\right)-N \operatorname{Vol}(X) .
$$

Furthermore, if $\chi \in \mathcal{W}^{-} \cup \mathcal{W}_{M}^{+}, M \geq 1$, then $\mathcal{E}_{\chi}(X, \omega)$ is convex.

For the definition of the weights $\mathcal{W}^{-}, \mathcal{W}_{M}^{+}$and further properties of finite energy classes we refer to Section 2.3. Convexity of $\mathcal{E}_{\chi}(X, \omega)$ was already established GZ1] in case $\chi \in \mathcal{W}_{M}^{+}, M \geq 1$. The case $\chi \in \mathcal{W}^{-}$was conjectured in [BEGZ, Remark 2.16].

Next we turn to a characterization of $\mathcal{E}(X, \omega)$ in terms of singularity types. For $u, v \in \operatorname{PSH}(X, \omega)$ we say that $u$ and $v$ have the same singularity type if there exists $c \in(0, \infty)$ such that

$$
u-c \leq v \leq u+c .
$$

This induces an equivalence relation on $\operatorname{PSH}(X, \omega)$ and we denote by $[u]$ the class of a representative $u \in \operatorname{PSH}(X, \omega)$.

Suppose now that $u_{0}, u_{1} \in \operatorname{PSH}(X, \omega)$. In [RWN] the envelope of $u_{0}$ with respect to the singularity type of $u_{1}$ was introduced in the following manner:

$$
P_{\left[u_{1}\right]}\left(u_{0}\right)=\operatorname{usc}\left(\lim _{c \rightarrow \infty} P\left(u_{1}+c, u_{0}\right)\right) .
$$

If $u_{0}$ is bounded and $u_{1} \not \equiv-\infty$ then $P_{\left[u_{1}\right]}\left(u_{0}\right) \not \equiv-\infty$. As a consequence of our first result, we see that this is also true when $u_{0}, u_{1} \in \mathcal{E}(X, \omega)$.

The functions of $\mathcal{E}(X, \omega)$ can be unbounded, but their singularities are mild. In particular, by Corollary 1.8 [GZ1], at any $x \in X$ the Lelong number of $v$ is zero. However, as noted in [GZ1], the converse is false. Membership in $\mathcal{E}(X, \omega)$ can nevertheless be characterized in terms of envelopes:

Theorem ([Da2 $)$. Suppose $u_{0} \in \operatorname{PSH}(X, \omega) \cap C(X)$ and $u_{1} \in \operatorname{PSH}(X, \omega)$. Then $u_{1} \in$ $\mathcal{E}(X, \omega)$ if and only if

$$
P_{\left[u_{1}\right]}\left(u_{0}\right)=u_{0}
$$

The condition $P_{\left[u_{1}\right]}\left(u_{0}\right)=u_{0}$ basically says that the singularity of $u_{1}$ is so mild that it is undetectable under envelope construction (9). It is also easily seen to imply that all Lelong numbers of $u_{1}$ are zero [Da2]. A question that immediately arises is weather the technical condition $u_{0} \in C(X)$ can be removed in the above result. Our next theorem, for which we will find other uses as well, says that this is indeed the case, what is more, $u_{0}$ need not even be bounded.

Theorem 4 (Theorem 4.3). Suppose $u_{0} \in \mathcal{E}(X, \omega)$ and $u_{1} \in \operatorname{PSH}(X, \omega)$. Then $u_{1} \in$ $\mathcal{E}(X, \omega)$ if and only if

$$
P_{\left[u_{1}\right]}\left(u_{0}\right)=u_{0}
$$


We return now to weak geodesics. Given $u_{0}, u_{1} \in \operatorname{PSH}(X, \omega)$, for the weak geodesic $t \rightarrow u_{t}$ defined in (3) it may easily happen that $u \equiv-\infty$. If this is not the case, one wonders in what sense $u_{t}$ approaches $u_{0}, u_{1}$ as $t \rightarrow 0,1$. To investigate these questions we revisit the notion of capacity. As introduced by Kołodziej [K], the Monge-Ampère capacity of a Borel set $B \subset X$ is defined by the formula

$$
\operatorname{Cap}(B)=\sup \left\{\int_{B}(\omega+i \partial \bar{\partial} u)^{n} \mid u \in \operatorname{PSH}(X, \omega), 0<u<1\right\} .
$$

For a sequence $\left\{v_{k}\right\}_{k \in \mathbb{N}} \subset \operatorname{PSH}(X, \omega)$ we say that $v_{k} \rightarrow v \in \operatorname{PSH}(X, \omega)$ in capacity if for any $\varepsilon>0$ we have

$$
\lim _{k \rightarrow \infty} \operatorname{Cap}\left(\left\{x \in X|| v_{k}(x)-v(x) \mid>\varepsilon\right\}\right)=0 .
$$

We note that convergence in capacity is stronger then convergence in $L^{1}(X)$ and is perhaps the strongest notion of convergence for unbounded plurisubharmonic functions. For an extensive study of capacities on compact Kähler manifolds we refer to GZ2].

We say that $u_{0}, u_{1} \in \operatorname{PSH}(X, \omega)$ can be connected with a weak geodesic if the curve $t \rightarrow u_{t}$ defined in (3) satisfies $u \not \equiv \infty$ and $\lim _{t \rightarrow 0,1} u_{t}=u_{0,1}$ in capacity. It is not readily clear what pairs of potentials $\left(u_{0}, u_{1}\right)$ can be joined by a weak geodesic. Our next result connects this issue with properties of the different types of envelopes we introduced.

Theorem 5 (Theorem 5.2). Suppose $u_{0}, u_{1} \in \operatorname{PSH}(X, \omega)$. Then for the curve $t \rightarrow u_{t}$ defined in (3) we have:

(i) $u \not \equiv-\infty$ if and only if $P\left(u_{0}, u_{1}\right) \not \equiv-\infty$.

(ii) $\lim _{t \rightarrow 0} u_{t}=u_{0}$ in capacity if and only if $P_{\left[u_{1}\right]}\left(u_{0}\right)=u_{0}$.

(iii) $\lim _{t \rightarrow 1} u_{t}=u_{1}$ in capacity if and only if $P_{\left[u_{0}\right]}\left(u_{1}\right)=u_{1}$.

As a trivial consequence of the last theorem we observe that if $u_{0}$ and $u_{1}$ have the same singularity type, then $u_{0}$ and $u_{1}$ can be connected with a weak geodesic. However, using (7), a more precise result can be obtained when $u_{0}, u_{1}$ are elements of finite energy classes:

Theorem 6 (Corollary [5.4). Suppose $\chi \in \mathcal{W}^{-} \cup \mathcal{W}_{M}^{+}, M \geq 1$ and $u_{0}, u_{1} \in \mathcal{E}_{\chi}(X, \omega)$. Then for the curve $t \rightarrow u_{t}$ defined in (3) we have:

(i) $u_{t} \in \mathcal{E}_{\chi}(X, \omega)$ for all $t \in(0,1)$. More precisely, if $N \in \mathbb{R}$ is such that $\chi \circ u_{0}, \chi \circ u_{1} \leq$ $N$ then we have:

$$
E_{\chi}\left(u_{t}\right) \geq C\left(E_{\chi}\left(u_{0}\right)+E_{\chi}\left(u_{1}\right)-N \operatorname{Vol}(X)\right),
$$

where $C$ only depends on $M$ and $\operatorname{dim} X$.

(ii) $\lim _{t \rightarrow t_{0}} u_{t}=u_{t_{0}}$ in capacity for any $t_{0} \in[0,1]$.

Given $u_{0} \in \operatorname{PSH}(X, \omega)$, one might want to find all $u_{1} \in \operatorname{PSH}(X, \omega)$ such that $u_{0}$ and $u_{1}$ can be connected with a weak geodesic. As a consequence of Theorem 4 and Theorem 5. for $u_{0} \in \mathcal{E}(X, \omega)$ we can provide an answer:

Corollary 7 (Corollary 5.5). Suppose $u_{0} \in \mathcal{E}(X, \omega)$ and $u_{1} \in \operatorname{PSH}(X, \omega)$. Then $u_{0}$ can be connected to $u_{1}$ with a weak geodesic if and only if $u_{1} \in \mathcal{E}(X, \omega)$. 
Further applications and possible future directions. Building on the techniques of this paper, in [Da3] we explore the Orlicz-Finsler geometry of $\mathcal{H}$, that is intimately tied together with the finite energy classes $\mathcal{E}_{\chi}(X, \omega)$ for $\chi \in \mathcal{W}_{M}^{+}$. In addition to this, we also prove that convergence with respect to the path length metric $\tilde{d}$ can be characterized using very concrete terms. Indeed, we show that there exists $C>1$ such that for any $u_{0}, u_{1} \in \mathcal{E}^{2}(X, \omega)$ we have:

$$
\frac{1}{C} \tilde{d}\left(u_{0}, u_{1}\right)^{2} \leq \int_{X}\left(u_{0}-u_{1}\right)^{2} \omega_{u_{0}}^{n}+\int_{X}\left(u_{0}-u_{1}\right)^{2} \omega_{u_{1}}^{n} \leq C \tilde{d}\left(u_{0}, u_{1}\right)^{2} .
$$

Further applications of the techniques developed here are explored in [DH], where we carry out a divergence analysis of the Kähler-Ricci flow in terms of the metric $\tilde{d}$. We also construct destabilizing geodesic rays weakly asymptotic to diverging Kähler-Ricci flow trajectories, with this partially verifying a folklore conjecture.

Convergence of metrics with respect to the Calabi metric is equivalent to the $L^{1}$ convergence of their volume densities [CR]. Though seemingly unrelated, in a future publication we hope to compare the geometry/topology of the Calabi metric with that of the the Mabuchi metric, as proposed in $\mathrm{CR}$.

In this paper we study extensively the end point problem for geodesics segments inside the metric completion of $\mathcal{H}$. Following the sequence of works initiated in [RZ], it would be also natural to investigate the analogous initial value problem in the general context of the metric completion.

Acknowledgements. I would like to thank L. Lempert and Y. Rubinstein for their suggestions on how to improve the presentation of the paper and for their support during the years. I would also like to thank M. Jonsson for useful discussions, in particular for noticing that Theorem 3 answers a question raised in BEGZ. I also profited from discussions with V. Guedj, G.K. Misiolek, and G. Székelyhidi. The fact that the metric completion of $\mathcal{H}$ is non-positively curved has been explored recently by J. Streets in connection with the Calabi flow ([St1, St2]). We thank him for bringing to our attention his interesting papers.

Organization. In Section 2 we recall preliminary material about finite energy classes and geodesics that we will need the most. We prove Theorem 3 in Section 3, Theorem 4 in Section 4 and Theorems 5 and 6 in Section 5. The proof of Theorem 1 is given in Sections 6-9. Readers interested only in the metric completion problem, should read Section 3, then skip ahead to the proof of Theorem 6(i) and from here proceed to Sections 6-9.

\section{Preliminaries}

\subsection{Distances in the Metric Space $(\mathcal{H}, d)$}

We summarize some of the properties of the metric space $(\mathcal{H}, d)$ that we will need later. This short section is based entirely on the findings of [C]. As in the introduction, given a smooth curve $[0,1] \ni t \rightarrow \alpha_{t} \in \mathcal{H}$, we define its length by the formula:

$$
l(\alpha)=\int_{0}^{1} \sqrt{\int_{X} \dot{\alpha}_{t}^{2}\left(\omega+i \partial \bar{\partial} \alpha_{t}\right)^{n}} d t .
$$


The distance $d\left(u_{0}, u_{1}\right)$ between two points $u_{0}, u_{1} \in \mathcal{H}$ is defined as the infimum of the length of smooth curves joining $u_{0}, u_{1}$. According to [C], $d$ is a metric on $\mathcal{H}$.

As noted earlier, in general there is no geodesic joining $u_{0}, u_{1}$ in $\mathcal{H}$, we only have a weak geodesic segment $[0,1] \ni t \rightarrow u_{t} \in \mathcal{H}_{\Delta}=\left\{u \in \operatorname{PSH}(X, \omega) \mid \Delta u \in L^{\infty}(X)\right\}$ solving (1) in the Bedford-Taylor sense, having bounded Laplacian. To address this technical inconvenience, X. X. Chen introduced the notion of $\varepsilon$-geodesics. An $\varepsilon$-geodesic joining $u_{0}, u_{1}$ is a smooth curve $[0,1] \ni t \rightarrow u_{t}^{\varepsilon} \in \mathcal{H}$ connecting $u_{0}, u_{1}$, that satisfies the equation:

$$
\left(\ddot{u}_{t}^{\varepsilon}-\frac{1}{2}\left\langle\nabla \dot{u}_{t}^{\varepsilon}, \nabla \dot{u}_{t}^{\varepsilon}\right\rangle\right)\left(\omega+i \partial \bar{\partial} u_{t}^{\varepsilon}\right)^{n}=\varepsilon \omega^{n}, t \in(0,1) .
$$

Chen proved the existence of such curve for any $\varepsilon>0$, along with the formula:

$$
d\left(u_{0}, u_{1}\right)=\lim _{\varepsilon \rightarrow 0} l\left(u^{\varepsilon}\right) .
$$

It is also proved that there exists $C>0$ independent of $\varepsilon$ such that

$$
\left\|\Delta u^{\varepsilon}\right\|_{L^{\infty}([0,1] \times X)} \leq C .
$$

Still, one would like to relate $d\left(u_{0}, u_{1}\right)$ directly to the weak geodesic $[0,1] \ni t \rightarrow u_{t} \in \mathcal{H}_{\Delta}$ joining $u_{0}, u_{1}$. For this we analyze (13) more closely. We have that

$$
l\left(u^{\varepsilon}\right)=\int_{0}^{1} \sqrt{E^{\varepsilon}(t)} d t
$$

where $E^{\varepsilon}(t)=\int_{X} \dot{u}_{t}^{\varepsilon^{2}}\left(\omega+i \partial \bar{\partial} u_{t}^{\varepsilon}\right)^{n}$. Using (12), one can easily compute that

$$
\frac{d E^{\varepsilon}(t)}{d t}=2 \varepsilon \int \dot{u}_{t}^{\varepsilon} \omega^{n}
$$

hence $\left|\dot{E}^{\varepsilon}(t)\right| \leq 2 \varepsilon\left\|\dot{u}_{t}^{\varepsilon}\right\|_{L^{\infty}(X)}, t \in[0,1]$. Estimate (14) further implies that

$$
\left|\dot{E}^{\varepsilon}(t)\right| \leq 2 \varepsilon C, t \in[0,1]
$$

It follows from this that for any $t_{0} \in[0,1]$ we have $\left|l\left(u^{\varepsilon}\right)-\sqrt{E^{\varepsilon}\left(t_{0}\right)}\right|=\mid \int_{0}^{1}\left(\sqrt{E^{\varepsilon}(t)}-\right.$ $\left.\sqrt{E^{\varepsilon}\left(t_{0}\right)}\right) d t \mid \leq \sqrt{2 \varepsilon C}$. This coupled with (13) implies that

$$
d\left(u_{0}, u_{1}\right)=\sqrt{\lim _{\varepsilon \rightarrow 0} E^{\varepsilon}\left(t_{0}\right)}
$$

It follows from the comparison principle ([B11, Theorem 6.4]) that our $\varepsilon$-geodesics $u^{\varepsilon}$ increase to the weak geodesic $u$ joining $u_{0}, u_{1}$, in particular $u_{t_{0}}^{\varepsilon}$ increases to $u_{t_{0}}$. BedfordTaylor theory implies now that $\left(\omega+i \partial \bar{\partial} u_{t_{0}}^{\varepsilon}\right)^{n} \rightarrow\left(\omega+i \partial \bar{\partial} u_{t_{0}}\right)^{n}$ in the weak sense of measures. By (14), using the Arzelà-Ascoli theorem it follows that we can find a subsequence of $u_{t_{0}}^{\varepsilon}$ (again denoted $u_{t_{0}}^{\varepsilon}$ ) such that $C(X) \ni \dot{u}_{t_{0}}^{\varepsilon} \rightarrow \dot{u}_{t_{0}} \in C(X)$ uniformly. The last two statements imply the following formula, again from $[\mathrm{C}]$ :

$$
d\left(u_{0}, u_{1}\right)=\sqrt{\lim _{\varepsilon \rightarrow 0} E^{\varepsilon}\left(t_{0}\right)}=\sqrt{\int_{X} \dot{u}_{t_{0}}^{2}\left(\omega+i \partial \bar{\partial} u_{t_{0}}\right)^{n}} .
$$

In Section 7 we will revisit (15) in a more general setting. 


\subsection{Bounded Weak Geodesic Segments and Envelopes}

X. X. Chen's notion of weak geodesic can be generalized to construct weak geodesic segments connecting points of $\mathcal{H}_{0}=\operatorname{PSH}(X, \omega) \cap L^{\infty}(X)$. Following Berndtssson, we recall how this argument works.

As before, let $S \subset \mathbb{C}$ be the strip $\{0<\operatorname{Re} s<1\}$ and $\tilde{\omega}$ be the pullback of $\omega$ to the product $S \times X$. As argued in [Br, Section 2.1], for $u_{0}, u_{1} \in \mathcal{H}_{0}$ the following Dirichlet problem has a unique solution:

$$
\begin{aligned}
& u \in \operatorname{PSH}(S \times X, \tilde{\omega}) \cap L^{\infty}(S \times X) \\
& (\tilde{\omega}+i \partial \bar{\partial} u)^{n+1}=0 \\
& u(t+i r, x)=u(t, x) \forall x \in X, t \in(0,1), r \in \mathbb{R} \\
& \lim _{t \rightarrow 0,1} u(t, x)=u_{0,1}(x), \forall x \in X .
\end{aligned}
$$

Since the solution to this equation is invariant in the imaginary direction, we denote it by $(0,1) \ni t \rightarrow u_{t} \in \mathcal{H}_{0}$ and call it the weak geodesic segment joining $u_{0}$ and $u_{1}$. Unsurprisingly, when $u_{0}, u_{1} \in \mathcal{H}$ this is the same curve as the weak geodesic $t \rightarrow u_{t}$ mentioned in the previous section. Fittingly, a curve $(0,1) \ni t \rightarrow v_{t} \in \operatorname{PSH}(X, \omega)$ is called a subgeodesic if $v(s, x):=v_{\text {Res }}(x) \in \operatorname{PSH}(S \times X, \tilde{\omega})$.

As a reminder, let us mention that the solution $u$ is constructed as the upper envelope

$$
u=\sup _{v \in \mathcal{S}} v
$$

where $\mathcal{S}$ is the following set of weak subgeodesics:

$$
\mathcal{S}=\left\{(0,1) \ni t \rightarrow v_{t} \in \operatorname{PSH}(X, \omega) \text { is a subgeodesic with } \lim _{t \rightarrow 0,1} v_{t} \leq u_{0,1}\right\} .
$$

Berndtsson also proved that $u$ is Lipschitz in the $t$-variable:

$$
\left\|\frac{\partial u}{\partial t}\right\|_{L^{\infty}(X)} \leq\left\|u_{0}-u_{1}\right\|_{L^{\infty}(X)} .
$$

Now we introduce the Legendre transform $\mathbb{R} \ni \tau \rightarrow u_{\tau}^{*} \in \operatorname{PSH}(X, \omega)$ of the weak geodesic segment $t \rightarrow u_{t}$ defined in (17):

$$
u_{\tau}^{*}=\inf _{t \in(0,1)}\left(u_{t}-\tau t\right) .
$$

The fact that $u_{\tau}^{*} \in \operatorname{PSH}(X, \omega)$ is guaranteed by Kiselman's minimum principle. The following identity is a particular case of a formula discovered in [DR], where some of its applications are studied:

$$
u_{\tau}^{*}=P\left(u_{0}, u_{1}-\tau\right)
$$

The proof of this identity is quite elementary. On the one hand $P\left(u_{0}, u_{1}-\tau\right) \leq u_{\tau}^{*}$ follows from (17), as the subgeodesic $t \rightarrow P\left(u_{0}, u_{1}-\tau\right)+t \tau$ is included in $\mathcal{S}$. On the other hand, we clearly have $u_{\tau}^{*} \leq \min \left(u_{0}, u_{1}-\tau\right)$, hence the definition of $P\left(u_{0}, u_{1}-\tau\right)$ implies the direction $u_{\tau}^{*} \leq P\left(u_{0}, u_{1}-\tau\right)$.

The regularity of envelopes of the type $P\left(u_{0}, u_{1}\right)$ will play an important role in this work. This is studied in [DR] and we mention here the following particular case of interest: 
Theorem 2.1 ([DR] $)$. If $u_{0}, u_{1} \in \mathcal{H}_{\Delta}$ then $P\left(u_{0}, u_{1}\right) \in \mathcal{H}_{\Delta}$.

A quick proof for this theorem can be given using the already existent regularity theory of weak geodesic segments [DR, Remark 4.3].

Proposition 2.2. Given $u_{0}, u_{1} \in \mathcal{H}_{\Delta}$ we introduce $\Lambda_{u_{0}}=\left\{P\left(u_{0}, u_{1}\right)=u_{0}\right\}$ and $\Lambda_{u_{1}}=$ $\left\{P\left(u_{0}, u_{1}\right)=u_{1}\right\}$. Then the following partition formula holds for the Monge-Ampère measure of $P\left(u_{0}, u_{1}\right)$ :

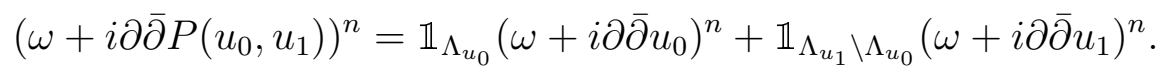

Proof. From [BT, Corollary 9.2] it follows that $\left(\omega+i \partial \bar{\partial} P\left(u_{0}, u_{1}\right)\right)^{n}$ is concentrated on the coincidence set $\Lambda_{u_{0}} \cup \Lambda_{u_{1}}$. Having bounded Laplacian implies that all second order partials of $P\left(u_{0}, u_{1}\right)$ are in any $L^{p}(X), p<\infty$. It follows from GT, Chapter 7, Lemma 7.7] that on $\Lambda_{u_{0}}$ all the second order partials of $P\left(u_{0}, u_{1}\right)$ and $u_{0}$ agree a.e. and an analogous statement holds on $\Lambda_{u_{1}}$. Hence, using [Bl4, Proposition 2.1.6] one can write:

$$
\begin{aligned}
& \left(\omega+i \partial \bar{\partial} P\left(u_{0}, u_{1}\right)\right)^{n}=\mathbb{1}_{\Lambda_{u_{0}} \cup \Lambda_{u_{1}}}\left(\omega+i \partial \bar{\partial} P\left(u_{0}, u_{1}\right)\right)^{n} \\
& =\mathbb{1}_{\Lambda_{u_{0}}}\left(\omega+i \partial \bar{\partial} u_{0}\right)^{n}+\mathbb{1}_{\Lambda_{u_{1} \backslash \Lambda_{u_{0}}}}\left(\omega+i \partial \bar{\partial} u_{1}\right)^{n},
\end{aligned}
$$

finishing the proof.

The partition formula (20) is at the core of most theorems in this work. Interestingly, it does not hold even in the slightly more general case $u_{0}, u_{1} \in \mathcal{H}_{0,1}$. For a counterexample suppose $\operatorname{dim} X=1$ and $g_{x}$ is the $\omega$-Green function with pole at $x \in X$. Such function is characterized by the property $\int_{X} g_{x} \omega=0$ and $\omega+i \partial \bar{\partial} g_{x}=\delta_{x}$. We choose $u_{0}=\max \left\{g_{x}, 0\right\}$ and $u_{1}=0$. In this case $P\left(u_{0}, u_{1}\right)=0, \Lambda_{u_{0}}=\left\{g_{x} \leq 0\right\}$ and $\Lambda_{u_{1}}=X \backslash \Lambda_{u_{0}} \neq \emptyset$. As $\operatorname{Vol}(X)=\int_{\Lambda_{u_{0}}}\left(\omega+i \partial \bar{\partial} u_{0}\right)^{n}=\int_{X}\left(\omega+i \partial \bar{\partial} P\left(u_{0}, u_{1}\right)\right)^{n}$, it is seen that the right hand side of (20) has total integral greater the the left hand side, hence they can not equal.

Despite these difficulties, a one-sided generalization of this formula is still possible (see Proposition 3.3).

As the function $t \rightarrow \int_{\left\{u_{0} \leq u_{1}+t\right\}} \omega^{n}$ is increasing, by adding constants one can always arrange that $\Lambda_{u_{0}} \cap \Lambda_{u_{1}} \subset\left\{u_{0}=u_{1}\right\}$ has zero Lebesgue measure. Using this and the previous proposition, we can write down the following observation:

Remark 2.3. Given $u_{0}, u_{1} \in \mathcal{H}_{\Delta}$ for any $\tau \in \mathbb{R}$ outside a countable set we have that $\Lambda_{u_{0}} \cap \Lambda_{u_{1}+\tau}$ has Lebesgue measure zero, implying:

$$
\left(\omega+i \partial \bar{\partial} P\left(u_{0}, u_{1}+\tau\right)\right)^{n}=\mathbb{1}_{\Lambda_{u_{0}}}\left(\omega+i \partial \bar{\partial} u_{0}\right)^{n}+\mathbb{1}_{\Lambda_{u_{1}+\tau}}\left(\omega+i \partial \bar{\partial} u_{1}\right)^{n} .
$$

For more details on the regularity theory and more geometric properties of weak geodesic segments we refer to [CC], [B11], [CT], [Do], [H], [PS], [SZ], to name only a few articles in a very fast expanding literature.

\section{$2.3 \mathcal{E}(X, \omega)$ and Finite Energy Classes}

We recall here the most basic facts about the class $\mathcal{E}(X, \omega) \subset \operatorname{PSH}(X, \omega)$. Our treatment is very brief and we refer to [GZ1] and [BEGZ] for a more complete picture. For $v \in$ $\operatorname{PSH}(X, \omega)$, one can define the canonical cutoffs $v_{h} \in \mathcal{H}_{0}, h \in \mathbb{R}$ by the formula $v_{h}=$ 
$\max (-h, v)$. By an application of the comparison principle, it follows that the Borel measures

$$
\mathbb{1}_{\{v>-h\}}\left(\omega+i \partial \bar{\partial} v_{h}\right)^{n}
$$

are increasing in $h$. Even in $v$ is unbounded, one can still make sense of $(\omega+i \partial \bar{\partial} v)^{n}$ as the limit of these increasing measures:

$$
(\omega+i \partial \bar{\partial} v)^{n}=\lim _{h \rightarrow \infty} \mathbb{1}_{\{v>-h\}}\left(\omega+i \partial \bar{\partial} v_{h}\right)^{n} .
$$

With this definition, $(\omega+i \partial \bar{\partial} v)^{n}$ is called the non-pluripolar Monge-Ampère measure of $v$. It follows from (21) that

$$
\int_{X}(\omega+i \partial \bar{\partial} v)^{n} \leq \int_{X} \omega^{n}=\operatorname{Vol}(X)
$$

This brings us to the class $\mathcal{E}(X, \omega)$. By definition, $v \in \mathcal{E}(X, \omega)$ if

$$
\int_{X}(\omega+i \partial \bar{\partial} v)^{n}=\lim _{h \rightarrow \infty} \int_{X} \mathbb{1}_{\{v>-h\}}\left(\omega+i \partial \bar{\partial} v_{h}\right)^{n}=\operatorname{Vol}(X) .
$$

As shown in GZ1, one can think of $\mathcal{E}(X, \omega)$ as $\omega$-plurisubharmonic functions having finite weighted energy. Suppose $\chi: \mathbb{R} \cup\{-\infty\} \rightarrow \mathbb{R}$ is a continuous increasing function, with $\chi(0)=0$ and $\chi(-\infty)=-\infty$. Such $\chi$ is referred to as a weight. The set of all weights is denoted by $\mathcal{W}$. By definition, for $v \in \mathcal{E}(X, \omega)$ we have $v \in \mathcal{E}_{\chi}(X, \omega)$ if

$$
E_{\chi}(v)=\int_{X} \chi(v)(\omega+i \partial \bar{\partial} v)^{n}>-\infty .
$$

The following result says that the $\chi$-energy $E_{\chi}$ can be computed using approximation by the canonical cutoffs:

Proposition 2.4. [GZ1, Proposition 1.4] Suppose $u \in \mathcal{E}(X, \omega), \chi \in \mathcal{W}$ and $u_{k}=$ $\max (u,-k), k \in \mathbb{N}$. Then:

$$
E_{\chi}(u)>-\infty \text { if and only if } \inf _{k} E_{\chi}\left(u_{k}\right)=\inf _{k} \int_{X} \chi\left(u_{k}\right)\left(\omega+i \partial \bar{\partial} u_{k}\right)^{n}>-\infty .
$$

If the above condition holds then we additionally have $E_{\chi}(u)=\lim _{k \rightarrow \infty} E_{\chi}\left(u_{k}\right)$.

The two special classes of weights that we will be most interested are:

$$
\begin{aligned}
& \mathcal{W}^{-}=\{\chi \in \mathcal{W} \mid \chi \text { is convex and } \chi(t)=t, t \geq 0\}, \\
& \mathcal{W}_{M}^{+}=\left\{\chi \in \mathcal{W} \mid \chi \text { is concave and }\left|t \chi^{\prime}(t)\right| \leq M|\chi(t)|, t \leq 0\right\},
\end{aligned}
$$

where $M \geq 1$. The interest in the convex weights $\mathcal{W}^{-}$comes from the following fact GZ1, Proposition 2.2]:

$$
\mathcal{E}(X, \omega)=\left\{v \in \mathcal{E}_{\chi}(X, \omega) \mid \chi \in \mathcal{W}^{-}\right\} .
$$

Of special importance are the weights $\chi^{p}(t)=-(-t)^{p}, t \leq 0, p>0$ and the associated classes $\mathcal{E}^{p}(X, \omega)$. Observe that $\chi^{p} \in \mathcal{W}^{-}$for $p \leq 1$ and $\chi^{p} \in \mathcal{W}_{p}^{+}$for $p \geq 1$. The case $p=1$ class interpolates between convex and concave energy classes as it is most apparent that

$$
\mathcal{E}_{\nu}(X, \omega) \subset \mathcal{E}^{1}(X, \omega) \subset \mathcal{E}_{\chi}(X, \omega),
$$

for any $\nu \in \mathcal{W}_{M}^{+}$and $\chi \in \mathcal{W}^{-}$. The following result is sometimes called the "fundamental estimate": 
Proposition 2.5. GZ1, Lemma 2.3, Lemma 3.5] Suppose $\chi \in \mathcal{W}^{-} \cup \mathcal{W}_{M}^{+}, M \geq 1$. If $u, v \in \mathcal{E}_{\chi}(X, \omega)$ with $u \leq v \leq 0$ then

$$
E_{\chi}(u) \leq C E_{\chi}(v)
$$

where $C>0$ depends only on $M$ and the dimension of $X$.

If $\chi \in \mathcal{W}^{-} \cup \mathcal{W}_{M}^{+}, \quad M \geq 1$ then the $\chi$-energy has a very useful semi-continuity property:

Proposition 2.6. [GZ1, Proposition 5.6] Suppose $\chi \in \mathcal{W}^{-} \cup \mathcal{W}_{M}^{+}, M \geq 1$ and $\left\{u_{j}\right\}_{j \in \mathbb{N}} \subset$ $\mathcal{H}_{0}$ is a sequence decreasing to $u \in \operatorname{PSH}(X, \omega)$. If $\inf _{j} E_{\chi}\left(u_{j}\right)>-\infty$ then $u \in \mathcal{E}_{\chi}(X, \omega)$ and

$$
E_{\chi}(u) \geq \liminf _{j \rightarrow \infty} E_{\chi}\left(u_{j}\right) .
$$

Using the canonical cutoffs, the last two results imply the very important "monotonicity property":

Corollary 2.7. Suppose $\chi \in \mathcal{W}^{-} \cup \mathcal{W}_{M}^{+}, M \geq 1$. If $u \leq v$ and $u \in \mathcal{E}_{\chi}(X, \omega)$ then $v \in \mathcal{E}_{\chi}(X, \omega)$.

The usual continuity property of the Monge-Ampère operator from Bedford-Taylor theory is also preserved in this more general setting:

Proposition 2.8. [BEGZ, Theorem 2.17] Suppose $\left\{v_{k}\right\}_{k \in \mathbb{N}} \subset \mathcal{E}(X, \omega)$ decreases (increases a.e.) to $v \in \mathcal{E}(X, \omega)$. Then $\left(\omega+i \partial \bar{\partial} v_{k}\right)^{n} \rightarrow(\omega+i \partial \bar{\partial} v)^{n}$ weakly.

Lastly, let us mention the uniqueness theorem of S. Dinew. We will use this result multiple times.

Theorem 2.9. [Di] Suppose that $u, v \in \mathcal{E}(X, \omega)$ satisfy $(\omega+i \partial \bar{\partial} u)^{n}=(\omega+i \partial \bar{\partial} v)^{n}$. Then $u-v$ is constant.

\section{The Operator $(u, v) \rightarrow P(u, v)$ on $\mathcal{E}(X, \omega)$}

In this section we examine properties of the envelopes $P\left(u_{0}, u_{1}\right)$ when $u_{0}, u_{1}$ are from one the finite energy classes $\mathcal{E}_{\chi}(X, \omega)$. Before we start dealing with the general case, let us establish some preliminary results generalizing formulas at the end of Section 2.2. By Proposition 2.2, if $u_{0}, u_{1} \in \mathcal{H}$ then $\left(\omega+i \partial \bar{\partial} P\left(u_{0}, u_{1}\right)^{n}\right.$ only charges the coincidence set $\left\{P\left(u_{0}, u_{1}\right)=\min \left(u_{0}, u_{1}\right)\right\}$. It turns out that this statement extends to the case $u_{0}, u_{1} \in \mathcal{H}_{0}=\operatorname{PSH}(X, \omega) \cap L^{\infty}(X)$ :

Lemma 3.1. For $u_{0}, u_{1} \in \mathcal{H}_{0}$ we have

$$
\left(\omega+i \partial \bar{\partial} P\left(u_{0}, u_{1}\right)\right)^{n}\left(\left\{P\left(u_{0}, u_{1}\right)<\min \left(u_{0}, u_{1}\right)\right\}\right)=0 .
$$

Proof. By [BK] there exist decreasing sequences $u_{1}^{j}, u_{0}^{j} \in \mathcal{H}$ such that $u_{0}^{j} \searrow u_{0}$ and $u_{1}^{j} \searrow u_{1}$. By Proposition 2.2 this means that

$$
\int_{X}\left(\min \left(u_{0}^{j}, u_{1}^{j}\right)-P\left(u_{0}^{j}, u_{1}^{j}\right)\right)\left(\omega+i \partial \bar{\partial} P\left(u_{0}^{j}, u_{1}^{j}\right)\right)^{n}=0, j \in \mathbb{N} .
$$


Since $\min \left(u_{0}^{j}, u_{1}^{j}\right)=u_{0}^{j}+u_{1}^{j}-\max \left(u_{0}^{j}, u_{1}^{j}\right)$, and $\left\{u_{0}^{j}\right\}_{j \in \mathbb{N}},\left\{u_{1}^{j}\right\}_{j \in \mathbb{N}},\left\{\max \left(u_{0}^{j}, u_{1}^{j}\right)\right\}_{j \in \mathbb{N}}$ form decreasing sequences of uniformly bounded $\omega$-plurisubharmonic functions, by BedfordTaylor theory, we can take the limit in the above identity to obtain:

$$
\int_{X}\left(\min \left(u_{0}, u_{1}\right)-P\left(u_{0}, u_{1}\right)\right)\left(\omega+i \partial \bar{\partial} P\left(u_{0}, u_{1}\right)\right)^{n}=0 .
$$

From this the statement of the lemma follows.

With the next result, we take another step in generalizing Proposition 2.2.

Lemma 3.2. For $u_{0}, u_{1} \in \mathcal{H}_{0}$ we have

$$
\left(\omega+i \partial \bar{\partial} P\left(u_{0}, u_{1}\right)\right)^{n} \leq \mathbb{1}_{\left\{u_{0} \leq u_{1}\right\}}\left(\omega+i \partial \bar{\partial} u_{0}\right)^{n}+\mathbb{1}_{\left\{u_{1} \leq u_{0}\right\}}\left(\omega+i \partial \bar{\partial} u_{1}\right)^{n} .
$$

Proof. Let $u_{0}^{j}, u_{1}^{j}$ be strictly decreasing approximating sequences, as in the proof of the previous result. After taking the weak limit of measures, from Proposition 2.2 it follows that

$$
\left(\omega+i \partial \bar{\partial} P\left(u_{0}, u_{1}\right)\right)^{n} \leq\left(\omega+i \partial \bar{\partial} u_{0}\right)^{n}+\left(\omega+i \partial \bar{\partial} u_{1}\right)^{n}
$$

We next prove that

$$
\mathbb{1}_{\left\{u_{0}<u_{1}\right\}}\left(\omega+i \partial \bar{\partial} P\left(u_{0}, u_{1}\right)\right)^{n} \leq \mathbb{1}_{\left\{u_{0}<u_{1}\right\}}\left(\omega+i \partial \bar{\partial} u_{0}\right)^{n}
$$

First, we prove this estimate for smooth $u_{1}$. We can additionally assume without loss of generality that $\left\{u_{j}^{0}=u_{1}\right\}$ has Lebesgue measure zero $j \in \mathbb{N}$. In this case the set $\left\{u_{0}<u_{1}\right\}$ is open, more precisely, it is the union of the increasing open sets $\left\{u_{0}^{j}<u_{1}\right\}$. Let $\phi \in C^{\infty}(X)$ with supp $\phi \subset\left\{u_{0}<u_{1}\right\}$ and $\phi \geq 0$. Clearly, there exists $j_{0}$ such that supp $\phi \subset\left\{u_{0}^{j}<u_{1}\right\}$ for all $j \geq j_{0}$. Using Proposition 2.2 this implies that

$$
\int_{\left\{u_{0}<u_{1}\right\}} \phi\left(\left(\omega+i \partial \bar{\partial} u_{0}^{j}\right)^{n}-\left(\omega+i \partial \bar{\partial} P\left(u_{0}^{j}, u_{1}\right)\right)^{n}\right) \geq 0, j \geq j_{0} .
$$

Letting $j \rightarrow \infty$ we arrive at

$$
\int_{\left\{u_{0}<u_{1}\right\}} \phi\left(\left(\omega+i \partial \bar{\partial} u_{0}\right)-\left(\omega+i \partial \bar{\partial} P\left(u_{0}, u_{1}\right)\right)^{n}\right) \geq 0, j \geq j_{0} .
$$

This implies (25) in the case $u_{1}$ is smooth. Now we treat the general case. Observe that the set $\left\{u_{0}<u_{1}\right\}$ is contained in the intersection of the decreasing open sets $\left\{u_{0}<u_{1}^{j}\right\}, j \in \mathbb{N}$. In particular, by what we just proved,

$$
\mathbb{1}_{\left\{u_{0}<u_{1}\right\}}\left(\omega+i \partial \bar{\partial} P\left(u_{0}, u_{1}^{j}\right)\right)^{n} \leq \mathbb{1}_{\left\{u_{0}<u_{1}\right\}}\left(\omega+i \partial \bar{\partial} u_{0}\right)^{n}, j \in \mathbb{N} .
$$

Since $P\left(u_{0}, u_{1}^{j}\right)$ decreases to $P\left(u_{0}, u_{1}\right)$ and $\left\{u_{0}<u_{1}\right\}$ is plurifine open, we can use [RWN, Lemma 3.5] to conclude that

$$
\mathbb{1}_{\left\{u_{0}<u_{1}\right\}}\left(\omega+i \partial \bar{\partial} P\left(u_{0}, u_{1}\right)\right)^{n} \leq \liminf _{j \rightarrow \infty} \mathbb{1}_{\left\{u_{0}<u_{1}\right\}}\left(\omega+i \partial \bar{\partial} P\left(u_{0}, u_{1}^{j}\right)\right)^{n} .
$$

Putting the last two estimates together we obtain (25). By (24) and (25) it results that:

$$
\begin{aligned}
\left(\omega+i \partial \bar{\partial} P\left(u_{0}, u_{1}\right)\right)^{n}= & \left(\mathbb{1}_{\left\{u_{0}<u_{1}\right\}}+\mathbb{1}_{\left\{u_{1}<u_{0}\right\}}+\mathbb{1}_{\left\{u_{0}=u_{1}\right\}}\right)\left(\omega+i \partial \bar{\partial} P\left(u_{0}, u_{1}\right)\right)^{n} \\
\leq & \mathbb{1}_{\left\{u_{0}<u_{1}\right\}}\left(\omega+i \partial \bar{\partial} u_{0}\right)^{n}+\mathbb{1}_{\left\{u_{1}<u_{0}\right\}}\left(\omega+i \partial \bar{\partial} u_{1}\right)^{n}+ \\
& +\mathbb{1}_{\left\{u_{0}=u_{1}\right\}}\left(\left(\omega+i \partial \bar{\partial} u_{0}\right)^{n}+\left(\omega+i \partial \bar{\partial} u_{1}\right)^{n}\right) .
\end{aligned}
$$

After rearranging terms we obtain the desired estimate. 
From the last two results we obtain an estimate that can be seen as a generalization of the partition formula in Proposition 2.2,

Proposition 3.3. For $u_{0}, u_{1} \in \mathcal{H}_{0}$ we have

$$
\left(\omega+i \partial \bar{\partial} P\left(u_{0}, u_{1}\right)\right)^{n} \leq \mathbb{1}_{\left\{P\left(u_{0}, u_{1}\right)=u_{0}\right\}}\left(\omega+i \partial \bar{\partial} u_{0}\right)^{n}+\mathbb{1}_{\left\{P\left(u_{0}, u_{1}\right)=u_{1}\right\}}\left(\omega+i \partial \bar{\partial} u_{1}\right)^{n}
$$

We now move on to proving energy estimates for envelopes of the type $P\left(u_{0}, u_{1}\right)$. As above, we deal with the bounded case first:

Lemma 3.4. Suppose $\chi \in \mathcal{W}, u_{0}, u_{1} \in \mathcal{H}_{0}$ and $N \in \mathbb{R}$ are such that $\chi \circ u_{0}, \chi \circ u_{1} \leq N$. Then

$$
E_{\chi}\left(P\left(u_{0}, u_{1}\right)\right) \geq E_{\chi}\left(u_{0}\right)+E_{\chi}\left(u_{1}\right)-N \operatorname{Vol}(X) .
$$

Proof. Since $P\left(u_{0}, u_{1}\right) \leq \min \left(u_{0}, u_{1}\right)$, it follows that $\chi \circ P\left(u_{0}, u_{1}\right) \leq N$.

$$
\begin{aligned}
& E_{\chi}\left(P\left(u_{0}, u_{1}\right)\right)-N \operatorname{Vol}(X)=\int_{X}\left(\chi\left(P\left(u_{0}, u_{1}\right)\right)-N\right)\left(\omega+i \partial \bar{\partial} P\left(u_{0}, u_{1}\right)\right)^{n} \\
& \quad \geq \int_{\left\{P\left(u_{0}, u_{1}\right)=u_{0}\right\}}\left(\chi\left(u_{0}\right)-N\right)\left(\omega+i \partial \bar{\partial} u_{0}\right)^{n}+\int_{\left\{P\left(u_{0}, u_{1}\right)=u_{1}\right\}}\left(\chi\left(u_{1}\right)-N\right)\left(\omega+i \partial \bar{\partial} u_{1}\right)^{n} \\
& \quad \geq \int_{X}\left(\chi\left(u_{0}\right)-N\right)\left(\omega+i \partial \bar{\partial} u_{0}\right)^{n}+\int_{X}\left(\chi\left(u_{1}\right)-N\right)\left(\omega+i \partial \bar{\partial} u_{1}\right)^{n} \\
& \quad=E_{\chi}\left(u_{0}\right)+E_{\chi}\left(u_{1}\right)-2 N \operatorname{Vol}(X) .
\end{aligned}
$$

where in the second line we have used (26) $)$.

Corollary 3.5. For $u_{0}, u_{1} \in \mathcal{E}(X, \omega)$, we have $P\left(u_{0}, u_{1}\right) \in \mathcal{E}(X, \omega)$. More precisely, if $u_{0}, u_{1} \in \mathcal{E}_{\chi}(X, \omega)$ for some $\chi \in \mathcal{W}^{-}$, then $P\left(u_{0}, u_{1}\right) \in \mathcal{E}_{\chi}(X, \omega)$.

Proof. Suppose $u_{0}, u_{1} \in \mathcal{E}_{\chi}(X, \omega)$ for some $\chi \in \mathcal{W}^{-}$. Let $N=\max \left(\sup _{X} \chi \circ u_{0}, \sup _{X} \chi \circ\right.$ $\left.u_{0}\right)$. If we apply the previous lemma to the canonical cutoffs $u_{0}^{k}, u_{1}^{k}$ we obtain:

$$
E_{\chi}\left(P\left(u_{0}^{k}, u_{1}^{k}\right)\right) \geq E_{\chi}\left(u_{0}^{k}\right)+E_{\chi}\left(u_{1}^{k}\right)-N \operatorname{Vol}(X) .
$$

Since $u_{0}, u_{1} \in \mathcal{E}_{\chi}(X, \omega)$ it follows that

$$
\begin{aligned}
\liminf _{k \rightarrow \infty} E_{\chi}\left(P\left(u_{0}^{k}, u_{1}^{k}\right)\right) & \geq \lim _{k \rightarrow \infty} E_{\chi}\left(u_{0}^{k}\right)+\lim _{k \rightarrow \infty} E_{\chi}\left(u_{1}^{k}\right)-N \operatorname{Vol}(X) \\
& =E_{\chi}\left(u_{0}\right)+E_{\chi}\left(u_{1}\right)-N \operatorname{Vol}(X),
\end{aligned}
$$

where we have used Proposition 2.4. Since $\chi \in \mathcal{W}^{-}$and $\inf _{k} E_{\chi}\left(P\left(u_{0}^{k}, u_{1}^{k}\right)\right)$ is bounded below, using Proposition 2.6 it follows that $P\left(u_{0}, u_{1}\right) \in \mathcal{E}_{\chi}(X, \omega)$.

If we start with $u_{0}, u_{1} \in \mathcal{E}(X, \omega)$, then by (23) it follows that there exists $\chi_{1}, \chi_{2} \in \mathcal{W}^{-}$ such that $u_{0} \in \mathcal{E}_{\chi_{1}}(X, \omega)$ and $u_{1} \in \mathcal{E}_{\chi_{2}}(X, \omega)$. Clearly $\chi=\max \left(\chi_{1}, \chi_{2}\right) \in \mathcal{W}^{-}$and $u_{0}, u_{1} \in \mathcal{E}_{\chi}(X, \omega)$. By our prior argument it follows that $P\left(u_{0}, u_{1}\right) \in \mathcal{E}_{\chi}(X, \omega) \subset \mathcal{E}(X, \omega)$, finishing the proof.

Finally, we generalize the estimate (27) for any $\chi \in \mathcal{W}$ and $u_{0}, u_{1} \in \mathcal{E}_{\chi}(X, \omega)$, with this proving Theorem 3 ; 
Theorem 3.6. If $\chi \in \mathcal{W}$ and $u_{0}, u_{1} \in \mathcal{E}_{\chi}(X, \omega)$, then $P\left(u_{0}, u_{1}\right) \in \mathcal{E}_{\chi}(X, \omega)$. More precisely, if $N \in \mathbb{R}$ is such that $\chi \circ u_{0}, \chi \circ u_{1} \leq N$, then we have:

$$
E_{\chi}\left(P\left(u_{0}, u_{1}\right)\right) \geq E_{\chi}\left(u_{0}\right)+E_{\chi}\left(u_{1}\right)-N \operatorname{Vol}(X) .
$$

Furthermore, if $\chi \in \mathcal{W}^{-} \cup \mathcal{W}_{M}^{+}, M \geq 1$, then $\mathcal{E}_{\chi}(X, \omega)$ is convex.

Proof. As both $E_{\chi}\left(u_{0}\right)$ and $E_{\chi}\left(u_{1}\right)$ are finite, we can use Lemma 3.4 and Proposition 2.4 to conclude:

$$
\liminf _{k \rightarrow \infty} E_{\chi}\left(P\left(u_{0}^{k}, u_{1}^{k}\right)\right) \geq E_{\chi}\left(u_{0}\right)+E_{\chi}\left(u_{1}\right)-N \operatorname{Vol}(X),
$$

where $u_{0}^{k}, u_{1}^{k}$ are the canonical cutoffs.

Fix $l \in \mathbb{N}$ and $v \in C(X)$ satisfying $v \geq \chi \circ P\left(u_{0}^{l}, u_{1}^{l}\right)$. As the sequence $P\left(u_{0}^{k}, u_{1}^{k}\right)$ decreases to $P\left(u_{0}, u_{1}\right)$ and $P\left(u_{0}, u_{1}\right) \in \mathcal{E}(X, \omega)$ by the previous result, we have $(\omega+$ $\left.i \partial \bar{\partial} P\left(u_{0}^{k}, u_{1}^{k}\right)\right)^{n} \rightarrow\left(\omega+i \partial \bar{\partial} P\left(u_{0}, u_{1}\right)\right)^{n}$ weakly. It follows that

$$
\int_{X} v\left(\omega+i \partial \bar{\partial} P\left(u_{0}, u_{1}\right)\right)^{n}=\lim _{k \rightarrow \infty} \int_{X} v\left(\omega+i \partial \bar{\partial} P\left(u_{0}^{k}, u_{1}^{k}\right)\right)^{n} \geq \liminf _{k \rightarrow \infty} E_{\chi}\left(P\left(u_{0}^{k}, u_{1}^{k}\right)\right) .
$$

As $\chi \circ P\left(u_{0}^{l}, u_{1}^{l}\right)$ is upper semi-continuous this implies:

$$
\int_{X} \chi\left(P\left(u_{0}^{l}, u_{1}^{l}\right)\right)\left(\omega+i \partial \bar{\partial} P\left(u_{0}, u_{1}\right)\right)^{n} \geq \liminf _{k \rightarrow \infty} E_{\chi}\left(P\left(u_{0}^{k}, u_{1}^{k}\right)\right) .
$$

Letting $l \rightarrow+\infty$, by the monotone convergence theorem one obtains

$$
E_{\chi}\left(P\left(u_{0}, u_{1}\right)\right) \geq \liminf _{k \rightarrow \infty} E_{\chi}\left(P\left(u_{0}^{k}, u_{1}^{k}\right)\right) .
$$

Putting (29) and (30) together, (28) follows. Finally, we have the estimate:

$$
P\left(u_{0}, u_{1}\right) \leq \min \left(u_{0}, u_{1}\right) \leq t u_{0}+(1-t) u_{1}, t \in[0,1]
$$

We just proved that $P\left(u_{0}, u_{1}\right) \in \mathcal{E}_{\chi}(X, \omega)$. If $\chi \in \mathcal{W}^{-} \cup \mathcal{W}_{M}^{+}, M \geq 1$ then Corollary 2.7 implies that $t u_{0}+(1-t) u_{1} \in \mathcal{E}_{\chi}(X, \omega), t \in[0,1]$.

We conclude from the above proof that in fact $\mathcal{E}_{\chi}(X, \omega)$ is convex for all weights $\chi$ that have the "monotonicity" property described in Corollary 2.7; if $u \in \mathcal{E}_{\chi}(X, \omega), v \in$ $\operatorname{PSH}(X, \omega)$ and $u \leq v$ then $v \in \mathcal{E}_{\chi}(X, \omega)$.

Finally, we notice that for arbitrary $u_{0}, u_{1} \in \operatorname{PSH}(X, \omega)$, it may easily happen that $P\left(u_{0}, u_{1}\right) \equiv-\infty$. If $X$ is $\mathbb{C} P^{1}$ with the Fubini-Study metric $\omega_{F S}$ and $u_{0}, u_{1}$ are $\omega_{F S}-$ Green functions with poles at different points, then clearly $P\left(u_{0}, u_{1}\right) \equiv-\infty$.

\section{The Operator $u \rightarrow P_{[u]}(v)$ on $\mathcal{E}(X, \omega)$}

In this short section we will prove Theorem 4 , Before we do this we need some preliminary results.

Lemma 4.1. Suppose $\chi \in \mathcal{W}$ and $u_{0}, u_{1} \in \mathcal{E}_{\chi}(X, \omega)$ with $u_{0}, u_{1}<0$. For any $c>0$ and $\phi \in C^{\infty}(X)$ with $\phi \geq 0$ we have

$$
\int_{X} \phi\left(\omega+i \partial \bar{\partial} P\left(u_{0}, u_{1}+c\right)\right)^{n} \leq \int_{X} \phi\left(\omega+i \partial \bar{\partial} u_{0}\right)^{n}+\frac{E_{\chi}\left(u_{1}\right) \sup _{X} \phi}{\chi(-c)} .
$$


Proof. Suppose $u_{0}^{j}, u_{1}^{j} \in \mathcal{H}_{0}$ are the canonical cutoffs decreasing to $u_{0}, u_{1}$. It follows that $P\left(u_{0}^{j}, u_{1}^{j}+c\right)$ decreases to $P\left(u_{0}, u_{1}+c\right)$. Theorem 3.6 implies that $P\left(u_{0}, u_{1}+c\right) \in \mathcal{E}_{\chi}(X, \omega)$ hence we have

$$
\int_{X} \phi\left(\omega+i \partial \bar{\partial} P\left(u_{0}, u_{1}+c\right)\right)^{n}=\lim _{j \rightarrow \infty} \int_{X} \phi\left(\omega+i \partial \bar{\partial} P\left(u_{0}^{j}, u_{1}^{j}+c\right)\right)^{n} .
$$

Using formula (26) we can write:

$$
\begin{aligned}
\int_{X} \phi(\omega+i & \left.\partial \bar{\partial} P\left(u_{0}^{j}, u_{1}^{j}+c\right)\right)^{n} \leq \\
& \leq \int_{\left\{P\left(u_{0}^{j}, u_{1}^{j}+c\right)=u_{0}^{j}\right\}} \phi\left(\omega+i \partial \bar{\partial} u_{0}^{j}\right)^{n}+\int_{\left\{P\left(u_{0}^{j}, u_{1}^{j}+c\right)=u_{1}^{j}+c\right\}} \phi\left(\omega+i \partial \bar{\partial} u_{1}^{j}\right)^{n} \\
& \leq \int_{X} \phi\left(\omega+i \partial \bar{\partial} u_{0}^{j}\right)^{n}+\int_{\left\{u_{1}^{j}<-c\right\}} \phi\left(\omega+i \partial \bar{\partial} u_{1}^{j}\right)^{n} \\
& \leq \int_{X} \phi\left(\omega+i \partial \bar{\partial} u_{0}^{j}\right)^{n}+\frac{\sup _{X} \phi}{\chi(-c)} \int_{\left\{u_{1}^{j}<-c\right\}} \chi\left(u_{1}^{j}\right)\left(\omega+i \partial \bar{\partial} u_{1}^{j}\right)^{n} \\
& \leq \int_{X} \phi\left(\omega+i \partial \bar{\partial} u_{0}^{j}\right)^{n}+\frac{\sup _{X} \phi}{\chi(-c)} \int_{X} \chi\left(u_{1}^{j}\right)\left(\omega+i \partial \bar{\partial} u_{1}^{j}\right)^{n} \\
& =\int_{X} \phi\left(\omega+i \partial \bar{\partial} u_{0}^{j}\right)^{n}+\frac{\sup _{X} \phi}{\chi(-c)} E_{\chi}\left(u_{1}^{j}\right) .
\end{aligned}
$$

Using Proposition 2.4, after taking the limit $j \rightarrow \infty$ in the above estimate we obtain the statement of the lemma.

Lemma 4.2. Suppose we have $u, v \in \operatorname{PSH}(X, \omega)$ satisfying $P_{[u]}(v) \not \equiv-\infty$. Then $\inf _{\left\{P_{[u]}(v)>-\infty\right\}}\left(v-P_{[u]}(v)\right)=0$.

Proof. Suppose $\inf _{\left\{P_{[u]}(v)>-\infty\right\}}\left(v-P_{[u]}(v)\right)>0$. Then there exists $\varepsilon>0$ such that $P_{[u]}(v) \leq v-\varepsilon$. This implies that $P(v, u+k) \leq v-\varepsilon, k \in \mathbb{N}$. From this it results that $P(v, u+k)=P(P(v, u+k), u+k) \leq P(v-\varepsilon, u+k)=P(v, u+k+\varepsilon)-\varepsilon, k \in \mathbb{N}$. Letting $k \rightarrow \infty$, we obtain $P_{[u]}(v) \leq P_{[u]}(v)-\varepsilon$, a contradiction.

Now we turn to the proof of Theorem 4;

Theorem 4.3. Suppose $u_{0} \in \mathcal{E}(X, \omega)$ and $u_{1} \in \operatorname{PSH}(X, \omega)$. Then $u_{1} \in \mathcal{E}(X, \omega)$ if and only if

$$
P_{\left[u_{1}\right]}\left(u_{0}\right)=u_{0}
$$

Proof. We can assume that $u_{0}, u_{1}<0$. Suppose $u_{1} \in \mathcal{E}(X, \omega)$. As explained in the proof of Corollary 3.5, one can find $\chi \in \mathcal{W}^{-}$such that $u_{0}, u_{1} \in \mathcal{E}_{\chi}(X, \omega)$. By Theorem 3.6 we have $P\left(u_{0}, u_{1}+k\right) \in \mathcal{E}_{\chi}(X, \omega), k \in \mathbb{N}$. By the continuity of the Monge-Ampère operator under increasing limits taken within the class $\mathcal{E}(X, \omega)$, we have $\left(\omega+i \partial \bar{\partial} P\left(u_{0}, u_{1}+k\right)\right)^{n} \rightarrow$ $\left(\omega+i \partial \bar{\partial} P_{\left[u_{1}\right]}\left(u_{0}\right)\right)^{n}$ weakly as $k \rightarrow \infty$. Using this, as $\chi(-\infty)=-\infty$, from Lemma 4.1 it follows that

$$
\left(\omega+i \partial \bar{\partial} P_{\left[u_{1}\right]}\left(u_{0}\right)\right)^{n} \leq\left(\omega+i \partial \bar{\partial} u_{0}\right)^{n} .
$$

Since both $u_{0}$ and $P_{\left[u_{1}\right]}\left(u_{0}\right)$ are in $\mathcal{E}_{\chi}(X, \omega)$, both of the measures above integrate to $\operatorname{Vol}(X)$ over $X$. Hence, we actually have equality in the above estimate. Now S. Dinew's uniqueness result (Theorem 2.9) and Lemma 4.2 yields that $P_{\left[u_{1}\right]}\left(u_{0}\right)=u_{0}$. 
For the other direction we use the results of [Da2]. To be more precise, let $\tilde{u}_{0} \in \mathcal{H}$ such that $\tilde{u}_{0} \geq u_{0}$ and $\tilde{u}_{0} \geq u_{1}$. We will argue that the geodesic ray $t \rightarrow v\left(\tilde{u}_{0}, u_{1}\right)_{t}$ constructed by the method of [Da2] is constant equal to $\tilde{u}_{0}$, thus implying that $u_{1} \in \mathcal{E}(X, \omega)$ [Da2, Theorem 2(iii)].

Let us recall that $(0, \infty) \ni t \rightarrow v\left(\tilde{u}_{0}, u_{1}\right)_{t} \in \mathcal{H}_{0}$ is a decreasing weak geodesic ray that is constructed as the increasing limit of the weak geodesic segments joining $\tilde{u}_{0}$ with $\max \left(u_{1}, \tilde{u}_{0}-k\right)$ parameterized by the segment $(0, k)$ [Da2, Section 4$]$. We also know that $v_{\infty}:=\lim _{t \rightarrow \infty} v\left(\tilde{u}_{0}, u_{1}\right)_{t} \geq u_{1}$.

From [Da2, Proposition 5.1] it follows that $P_{\left[v_{\infty}\right]}\left(\tilde{u}_{0}\right)=v_{\infty}$, hence

$$
u_{1} \leq P_{\left[u_{1}\right]}\left(\tilde{u}_{0}\right) \leq P_{\left[v_{\infty}\right]}\left(\tilde{u}_{0}\right)=v_{\infty}
$$

If follows from the method of constructing the ray $t \rightarrow v\left(\tilde{u}_{0}, u_{1}\right)_{t}$ that for any $\alpha \in$ $\operatorname{PSH}(X, \omega)$ with $u_{1} \leq \alpha \leq v_{\infty}$ we have $v\left(\tilde{u}_{0}, u_{1}\right)=v\left(\tilde{u}_{0}, \alpha\right)$. Using this, by the last estimate we have

$$
v\left(\tilde{u}_{0}, P_{\left[u_{1}\right]}\left(\tilde{u}_{0}\right)\right)_{t}=v\left(\tilde{u}_{0}, u_{1}\right)_{t},
$$

for any $t \in(0, \infty)$. Since $P_{\left[u_{1}\right]}\left(u_{0}\right) \leq P_{\left[u_{1}\right]}\left(\tilde{u}_{0}\right)$, we have $P_{\left[u_{1}\right]}\left(\tilde{u}_{0}\right) \in \mathcal{E}(X, \omega)$, hence by [Da2, Theorem 2(iii)] it follows that $t \rightarrow v\left(\tilde{u}_{0}, P_{\left[u_{1}\right]}\left(\tilde{u}_{0}\right)\right)_{t}$ is constant equal to $\tilde{u}_{0}$, implying that $t \rightarrow v\left(\tilde{u}_{0}, u_{1}\right)_{t}$ is constant as well. As mentioned in the beginning, applying Da2, Theorem 2(iii)] again, we obtain that $u_{1} \in \mathcal{E}(X, \omega)$.

\section{$5 \quad$ Weak Geodesic Segments in $\operatorname{PSH}(X, \omega)$}

Recall that given $u_{0}, u_{1} \in \operatorname{PSH}(X, \omega)$ and decreasing approximating sequences $u_{0}^{k}, u_{1}^{k} \in$ $\mathcal{H}$, we define the "candidate" weak geodesic $(0,1) \ni t \rightarrow u_{t} \in \operatorname{PSH}(X, \omega)$ joining $u_{0}$ and $u_{1}$ by the formula:

$$
u_{t}=\lim _{k \rightarrow+\infty} u_{t}^{k}, t \in(0,1),
$$

where $(0,1) \ni t \rightarrow u_{t}^{k} \in \mathcal{H}_{\Delta}$ are the weak geodesics joining $u_{0}^{k}, u_{1}^{k}$. We observe that this definition is independent of the choice of approximating sequences, as $u(s, x)=$ $u_{\operatorname{Re} s}(x) \in P S H(S \times X, \tilde{\omega})$ is the upper envelope of the family $\mathcal{S}$ :

$$
u=\sup _{v \in \mathcal{S}} v
$$

where $\mathcal{S}$ is the following set of subgeodesics:

$$
\mathcal{S}=\left\{(0,1) \ni t \rightarrow v_{t} \in \operatorname{PSH}(X, \omega) \text { is a subgeodesic with } \lim _{t \rightarrow 0,1} v_{t} \leq u_{0,1}\right\} .
$$

By (17) and (32) it is clear that when $u_{0}, u_{1} \in \mathcal{H}_{0}$ the weak geodesic defined in Section 2.2 and (31) are the same. Before we prove Theorem 5 we make and elementary observation about convex functions that will turn out to be quite useful.

Lemma 5.1. Given a bounded convex function $f:(0,1) \rightarrow \mathbb{R}$ we have

$$
\lim _{t \rightarrow 0} f(t)=\lim _{\tau \rightarrow-\infty} \inf _{t \in(0,1)}(f(t)-\tau t) .
$$


Proof. The estimate $\lim _{t \rightarrow 0} f(t) \geq \lim _{\tau \rightarrow-\infty} \inf _{t \in(0,1)}(f(t)-\tau t)$ is clear. Now we deal with the reverse estimate. If $f^{\prime}$ is bounded below on $(0,1 / 2]$ then we are done, since for negative enough $\tau$ the map $t \rightarrow f(t)-\tau t$ is increasing. If $f^{\prime}$ is unbounded on $(0,1 / 2]$, then for any $\tau<\min \left\{f^{\prime}(1 / 2), 0\right\}$ there exists $t_{\tau} \in(0,1 / 2)$ such that $\inf _{t \in(0,1)}(f(t)-\tau t)=$ $f\left(t_{\tau}\right)-\tau t_{\tau} \geq f\left(t_{\tau}\right)$. Clearly $t_{\tau} \rightarrow 0$ as $\tau \rightarrow-\infty$, hence $\lim _{\tau \rightarrow-\infty} \inf _{t \in(0,1)}(f(t)-\tau t) \geq$ $\lim _{t \rightarrow 0} f(t)$.

Theorem 5.2. Suppose $u_{0}, u_{1} \in \operatorname{PSH}(X, \omega), u_{0}, u_{1} \not \equiv-\infty$. Then for the curve $t \rightarrow u_{t}$ defined in (31) we have:

(i) $u \not \equiv-\infty$ if and only if $P\left(u_{0}, u_{1}\right) \not \equiv-\infty$.

(ii) $\lim _{t \rightarrow 0} u_{t}=u_{0}$ in capacity if and only if $P_{\left[u_{1}\right]}\left(u_{0}\right)=u_{0}$.

(iii) $\lim _{t \rightarrow 1} u_{t}=u_{1}$ in capacity if and only if $P_{\left[u_{0}\right]}\left(u_{1}\right)=u_{1}$.

Proof. We can suppose throughout the proof that $u_{0}, u_{1} \leq 0$. By approximating with a decreasing sequence of bounded weak geodesics, it is easily seen that formula (19) also holds for our possibly unbounded weak geodesic $t \rightarrow u_{t}$, that is

$$
P\left(u_{0}, u_{1}-\tau\right)=\inf _{t \in(0,1)}\left(u_{t}-\tau t\right)
$$

for all $\tau \in \mathbb{R}$. By convexity in the $t$-variable, $u \not \equiv-\infty$ if and only if $\inf _{t \in(0,1)} u_{t} \not \equiv-\infty$, which in turn is equivalent to $P\left(u_{0}, u_{1}\right) \not \equiv-\infty$. This proves (i).

Now we turn to the proof of (ii). We assume first that $P_{\left[u_{1}\right]}\left(u_{0}\right)=u_{0}$. Notice that, by formula (32), for any $c \in \mathbb{R}$ we have

$$
P\left(u_{0}, u_{1}+c\right)-c t \leq u_{t}, t \in(0,1)
$$

By convexity in the $t$ variable we can further write:

$$
P\left(u_{0}, u_{1}+c\right)-u_{0}-c t \leq u_{t}-u_{0} \leq t\left(u_{1}-u_{0}\right), t \in(0,1) .
$$

This implies that:

$$
\left\{\left|u_{t}-u_{0}\right|>\varepsilon\right\} \subset\left\{\left|P\left(u_{0}, u_{1}+c\right)-u_{0}\right|>\varepsilon-c t\right\} \cup\left\{\left|u_{1}-u_{0}\right|>\varepsilon / t\right\},
$$

for any $t \in(0,1)$. Since the capacity is subadditive we can write:

$$
\begin{aligned}
& \lim _{t \rightarrow 0} \operatorname{Cap}\left\{\left|u_{t}-u_{0}\right|>\varepsilon\right\} \leq \\
& \leq \limsup _{t \rightarrow 0} \operatorname{Cap}\left\{\left|P\left(u_{0}, u_{1}+c\right)-u_{0}\right|>\varepsilon-c t\right\}+\limsup _{t \rightarrow 0} \operatorname{Cap}\left\{\left|u_{1}-u_{0}\right|>\varepsilon / t\right\} \\
& \leq \operatorname{Cap}\left\{\left|P\left(u_{0}, u_{1}+c\right)-u_{0}\right|>\varepsilon / 2\right\}+\limsup _{t \rightarrow 0} \operatorname{Cap}\left\{\left|u_{1}-u_{0}\right|>\varepsilon / t\right\} .
\end{aligned}
$$

The last limit is zero as we have:

$$
\begin{aligned}
\limsup _{t \rightarrow 0} \operatorname{Cap}\left\{\left|u_{1}-u_{0}\right|>\varepsilon / t\right\} & \leq \limsup _{t \rightarrow 0} \operatorname{Cap}\left\{\left|u_{1}\right|+\left|u_{0}\right|>\varepsilon / t\right\} \\
& \leq \lim _{t \rightarrow 0}\left(\operatorname{Cap}\left\{u_{0}<-\varepsilon / 2 t\right\}+\operatorname{Cap}\left\{u_{1}<-\varepsilon / 2 t\right\}\right)=0 .
\end{aligned}
$$


Summing up we have

$$
\lim _{t \rightarrow 0} \operatorname{Cap}\left\{\left|u_{t}-u_{0}\right|>\varepsilon\right\} \leq \operatorname{Cap}\left\{\left|P\left(u_{0}, u_{1}+c\right)-u_{0}\right|>\varepsilon / 2\right\}, c \in \mathbb{R} .
$$

Our assumption implies that $P\left(u_{0}, u_{1}+c\right)$ increases to $u_{0}$ outside a set of zero capacity zero as $c \rightarrow+\infty$ (negligible sets are pluripolar). It is well known (using quasi-continuity for instance) that this implies

$$
\lim _{c \rightarrow+\infty} \operatorname{Cap}\left\{\left|P\left(u_{0}, u_{1}+c\right)-u_{0}\right|>\varepsilon / 2\right\}=0
$$

proving that $\lim _{t \rightarrow 0} u_{t}=u_{0}$ in capacity.

Now we prove the other direction. As noted earlier, $\lim _{t \rightarrow 0} u_{t}=u_{0}$ in capacity implies convergence in $L^{1}(X)$. Using convexity of $u$ in the $t$ variable again, we obtain that $u_{t}(x) \rightarrow u_{0}(x)$ for any $x \in X$ outside a set $E$ of Lebesgue measure 0 . Lemma 5.1 now implies that:

$$
u_{0}(x)=\lim _{\tau \rightarrow-\infty} \inf _{t \in(0,1)}\left(u_{t}(x)-\tau t\right), x \in X \backslash E .
$$

Additionally, formula (33) coupled with the fact that negligible sets are pluripolar tells us that for any $x \in X$ outside a set $C$ of capacity zero, we have

$$
\lim _{\tau \rightarrow-\infty} \inf _{t \in(0,1)}\left(u_{t}(x)-\tau t\right)=\lim _{\tau \rightarrow-\infty} P\left(u_{0}, u_{1}-\tau\right)(x)=P_{\left[u_{1}\right]}\left(u_{0}\right)(x) .
$$

Putting the last two formulas together we obtain that $u_{0}(x)=P_{\left[u_{1}\right]}\left(u_{0}\right)(x)$ for any $x \in X$ outside $E \cup C$. As $\operatorname{Cap}(C)=0$, it follows that $C$ has Lebesgue measure zero, implying that $E \cup C$ has Lebesgue measure zero as well. From this it results that $u_{0}=P_{\left[u_{1}\right]}\left(u_{0}\right)$ globally, finishing the proof of (ii). The proof of part (iii) is carried out the same way.

The next corollary can be extracted from the proof of the previous theorem.

Corollary 5.3. Suppose that $u_{0}, u_{1} \in P S H(X, \omega)$ and for the curve $t \rightarrow u_{t}$ defined in (31) we have $u \neq-\infty$. The following are equivalent:

(i) $P_{\left[u_{1}\right]}\left(u_{0}\right)=u_{0}$.

(ii) $\lim _{t \rightarrow 0} u_{t}=u_{0}$ in capacity.

(iii) $\lim _{t \rightarrow 0} u_{t}=u_{0}$ in $L^{1}(X)$.

(iv) $\lim _{t \rightarrow 0} u_{t}(x)=u_{0}(x)$ for any $x \in X$ outside a set of Lebesgue measure zero.

The analogous statement for limits at $t=1$ is also true.

Proof. By the previous theorem (i) and (ii) are equivalent. Clearly, (ii) implies (iii), which in turn implies (iv), since $u$ is convex in the $t$-variable. Finally, the direction (iv) $\rightarrow$ (i) follows from the last part of the proof of the previous theorem.

We say that $u_{0}, u_{1} \in \operatorname{PSH}(X, \omega), u_{0}, u_{1} \not \equiv-\infty$ can be connected with a weak geodesic if for the curve $t \rightarrow u_{t}$ defined in (31) we have $u \not \equiv-\infty$ and $\lim _{t \rightarrow 0,1} u_{t}=u_{0,1}$ in capacity. By putting together Theorem 3.6, Theorem 4.3 and Theorem 5.2 we obtain that for certain weights $\chi$, the elements of $\mathcal{E}_{\chi}(X, \omega)$ can always be connected with a weak geodesic segment passing through $\mathcal{E}_{\chi}(X, \omega)$. 
Corollary 5.4. Suppose $\chi \in \mathcal{W}^{-} \cup \mathcal{W}_{M}^{+}, M \geq 1$ and $u_{0}, u_{1} \in \mathcal{E}_{\chi}(X, \omega)$. Then for the curve $t \rightarrow u_{t}$ defined in (31) we have:

(i) $u_{t} \in \mathcal{E}_{\chi}(X, \omega)$ for all $t \in(0,1)$. More precisely, if $N \in \mathbb{R}$ satisfies $\chi \circ u_{0}, \chi \circ u_{1} \leq N$ then:

$$
E_{\chi}\left(u_{t}\right) \geq C\left(E_{\chi}\left(u_{0}\right)+E_{\chi}\left(u_{1}\right)-N \operatorname{Vol}(X)\right),
$$

where $C$ depends only on $M$ and $\operatorname{dim} X$.

(ii) $\lim _{t \rightarrow t_{0}} u_{t}=u_{t_{0}}$ in capacity for any $t_{0} \in[0,1]$.

Proof. Formula (33) implies that $u_{t} \geq P\left(u_{0}, u_{1}\right)$ for any $t_{0} \in[0,1]$. Part (i) follows now from Theorem 3.6. Proposition 2.5 and Proposition 2.7. When $t_{0}=0$ or $t_{0}=1$, part (ii) is a consequence of Theorem 4.3 and Theorem 5.2 (ii)(iii).

Let $t_{0} \in(0,1)$. By part (i) we have $u_{t_{0}} \in \mathcal{E}_{\chi}(X, \omega)$. Suppose $(0,1) \ni t \rightarrow v_{t} \in$ $\mathcal{E}_{\chi}(X, \omega)$ is the weak geodesic segment joining $u_{0}$ and $u_{t_{0}}$ defined by (31). By (32) it follows that $v_{t}=u_{t_{0} t}, t \in(0,1)$. Using what we just proved, we obtain that $\lim _{t} \nearrow_{t_{0}} u_{t}=$ $\lim _{t \nearrow_{1}} v_{t}=u_{t_{0}}$ in capacity. One deals with the right limit similarly to conclude that $\lim _{t \rightarrow t_{0}} u_{t}=u_{t_{0}}$ in capacity.

We remark that in concluding $u_{t} \in E_{\chi}(X, \omega)$ for all $t \in(0,1)$ we only used the fact that $\chi$ has the "monotonicity" property described in Proposition 2.7. Another application of Theorem 5.2 and Theorem 4.3 yields the last result of this section.

Corollary 5.5. Suppose $u_{0} \in \mathcal{E}(X, \omega)$ and $u_{1} \in \operatorname{PSH}(X, \omega)$. Then $u_{0}$ can be connected to $u_{1}$ with a weak geodesic if and only if $u_{1} \in \mathcal{E}(X, \omega)$.

\section{$6 \quad$ Extending the Mabuchi Metric to $\mathcal{E}^{2}(X, \omega)$}

Given $u_{0}, u_{1} \in \mathcal{E}^{2}(X, \omega)$ and decreasing approximating sequences $u_{0}^{k}, u_{1}^{k} \in \mathcal{H}$, we define the distance $\tilde{d}\left(u_{0}, u_{1}\right)$ by the formula:

$$
\tilde{d}\left(u_{0}, u_{1}\right)=\lim _{k \rightarrow \infty} d\left(u_{0}^{k}, u_{1}^{k}\right),
$$

The main result of this section is the following:

Theorem 6.1. $\left(\mathcal{E}^{2}(X, \omega), \tilde{d}\right)$ is a non-positively curved geodesic metric space extending $(\mathcal{H}, d)$, with geodesic segments joining $u_{0}, u_{1} \in \mathcal{E}^{2}(X, \omega)$ given by (31).

Proving completeness of $\left(\mathcal{E}^{2}(X, \omega), \tilde{d}\right)$ is left to a later section (Theorem 9.2). The proof of the above theorem will be split into a sequence of lemmas. Our first result is a well known estimate for the Mabuchi metric that will be used a lot:

Lemma $6.2([\mathrm{C}])$. Suppose $u, v \in \mathcal{H}$ with $u \leq v$. Then we have:

$$
\int_{X}(v-u)^{2}(\omega+i \partial \bar{\partial} v)^{n} \leq d(u, v)^{2} \leq \int_{X}(v-u)^{2}(\omega+i \partial \bar{\partial} u)^{n}
$$


Proof. Suppose $(0,1) \ni t \rightarrow w_{t} \in \mathcal{H}_{\Delta}$ is the weak geodesic segment joining $u$ and $v$. By (15) we have

$$
d(u, v)=\sqrt{\int_{X} \dot{w}_{0}^{2}(\omega+i \partial \bar{\partial} u)^{n}}=\sqrt{\int_{X} \dot{w}_{1}^{2}(\omega+i \partial \bar{\partial} v)^{n}} .
$$

Since $u \leq v$, we have that $u \leq w_{t}$, as follows from (17). Since $(t, x) \rightarrow w_{t}(x)$ is convex in the $t$-variable, it results that $0 \leq \dot{w}_{0} \leq v-u \leq \dot{w}_{1}$ and the lemma follows.

Lemma 6.3. Suppose $u \in \mathcal{E}^{2}(X, \omega)$ and $\left\{u_{k}\right\}_{k \in \mathbb{N}} \subset \mathcal{H}$ is a sequence decreasing to $u$. Then $d\left(u_{l}, u_{k}\right) \rightarrow 0$ as $l, k \rightarrow \infty$.

Proof. We can suppose that $l \leq k$. Then $u_{k} \leq u_{l}$, hence by the previous lemma we have:

$$
d\left(u_{l}, u_{k}\right)^{2} \leq \int_{X}\left(u_{k}-u_{l}\right)^{2}\left(\omega+i \partial \bar{\partial} u_{k}\right)^{n} .
$$

We clearly have $u-u_{l}, u_{k}-u_{l} \in \mathcal{E}^{2}\left(X, \omega+i \partial \bar{\partial} u_{l}\right)$ and $u-u_{l} \leq u_{k}-u_{l} \leq 0$. Hence, applying Proposition 2.5 for the class $\mathcal{E}^{2}\left(X, \omega+i \partial \bar{\partial} u_{l}\right)$ we obtain that

$$
d\left(u_{l}, u_{k}\right)^{2} \leq C \int_{X}\left(u-u_{l}\right)^{2}(\omega+i \partial \bar{\partial} u)^{n} .
$$

As $u_{l}$ decreases to $u \in \mathcal{E}^{2}(X, \omega)$, it follows from the dominated convergence theorem that $d\left(u_{l}, u_{k}\right) \rightarrow 0$ as $l, k \rightarrow \infty$.

Lemma 6.4. Given $u_{0}, u_{1} \in \mathcal{E}^{2}(X, \omega)$, the limit in (35) is finite and independent of the approximating sequences $u_{0}^{k}, u_{1}^{k} \in \mathcal{H}$. Additionally, if $u_{0}, u_{1} \in \mathcal{H}$, then $\tilde{d}\left(u_{0}, u_{1}\right)$ is equal to the Mabuchi distance $d\left(u_{0}, u_{1}\right)$.

Proof. By the triangle inequality and Lemma 6.3 we can write:

$$
\left|d\left(u_{0}^{l}, u_{1}^{l}\right)-d\left(u_{0}^{k}, u_{1}^{k}\right)\right| \leq d\left(u_{0}^{l}, u_{0}^{k}\right)+d\left(u_{1}^{l}, u_{1}^{k}\right) \rightarrow 0, l, k \rightarrow \infty,
$$

proving that $d\left(u_{0}^{k}, u_{1}^{k}\right)$ is indeed convergent.

Now we prove that the limit in (35) is independent of the choice of approximating sequences. Let $v_{0}^{l}, v_{1}^{l} \in \mathcal{H}$ be another approximating sequence. By adding small constants if necessary, we can arrange that all the sequences $u_{0}^{l}, u_{1}^{l}$ respectively $v_{0}^{l}, v_{1}^{l}$ are strictly decreasing to $u_{0}, u_{1}$.

Fixing $k$ for the moment, the sequence $\left\{\max \left\{u_{0}^{k+1}, v_{0}^{j}\right\}\right\}_{j \in \mathbb{N}}$ decreases pointwise to $u_{0}^{k+1}$. By Dini's lemma the convergence is uniform, hence there exists $j_{k} \in \mathbb{N}$ such that for any $j \geq j_{k}$ we have $v_{0}^{j}<u_{0}^{k}$. By repeating the same argument we can also assume that $v_{1}^{j}<u_{1}^{k}$ for any $j \geq j_{k}$. By the triangle inequality again

$$
\left|d\left(u_{0}^{j}, u_{1}^{j}\right)-d\left(v_{0}^{k}, v_{0}^{k}\right)\right| \leq d\left(u_{0}^{j}, v_{0}^{k}\right)+d\left(u_{1}^{j}, v_{1}^{k}\right), j \geq j_{k} .
$$

From (36) it follows that for $k$ big enough the quantities $d\left(u_{0}^{j}, v_{0}^{k}\right), d\left(u_{1}^{j}, v_{1}^{k}\right), j \geq j_{k}$ are arbitrarily small, hence $\tilde{d}\left(u_{0}, u_{1}\right)$ is independent of the choice of approximating sequences. We observe that this automatically implies that $\tilde{d}$ restricted to $\mathcal{H}$ is the Mabuchi metric. The triangle inequality for $\tilde{d}$ also follows. 
To conclude that $\tilde{d}$ is a metric on $\mathcal{E}^{2}(X, \omega)$ all we need is that $\tilde{d}\left(u_{0}, u_{1}\right)=0$ implies $u_{0}=u_{1}$. Before we prove this we make two elementary observations.

Lemma 6.5. Suppose $u_{0}, u_{1} \in \mathcal{H}_{0}$. Let $(0,1) \ni t \rightarrow u_{t} \in \mathcal{H}_{0}$ be the bounded weak geodesic joining $u_{0}$ and $u_{1}$. Then for any $\tau \in \mathbb{R}$ we have

$$
\left\{\dot{u}_{0} \geq \tau\right\}=\left\{P\left(u_{0}, u_{1}-\tau\right)=u_{0}\right\} .
$$

Proof. By (19) we have $\inf _{t \in[0,1]}\left(u_{t}-\tau\right)=P\left(u_{0}, u_{1}-\tau\right)$. Given $x \in X$, it follows that $P\left(u_{0}, u_{1}-\tau\right)(x)=u_{0}(x)$ if and only if $\inf _{t \in[0,1]}\left(u_{t}(x)-\tau\right)=u_{0}(x)$. The convexity in the $t$-variable implies that this last identity is equivalent to $\dot{u}_{0}(x) \geq \tau$.

The next result will serve a purpose similar to Lemma 4.2.

Lemma 6.6. Suppose $u, v \in \operatorname{PSH}(X, \omega)$ are from the same singularity class. Then $P(u, v) \not \equiv-\infty$, moreover either $P(u, v)=u$ or $\inf _{\{u>-\infty\}}(v-P(u, v))=0$.

Proof. As $u, v \in \operatorname{PSH}(X, \omega)$ are from the same singularity class it follows that for $c$ big enough $P(u, v) \geq u-c$. If $u \leq v$ then clearly $P(u, v)=u$.

Let us suppose that $u \not \leq v$ and define $l_{0}=\sup \{l \in \mathbb{R} \mid u+l \leq v\}$. From our assumptions it follows that $-\infty<l_{0}<0$. We have $u+l_{0} \leq u$ and $u+l_{0} \leq v$, hence $u+l_{0} \leq P(u, v)$. By the definition of $l_{0}$ we also have $\inf _{\{u>-\infty\}}\left(v-u-l_{0}\right)=0$. Since $u+l_{0} \leq P(u, v) \leq v$, it follows that $\inf _{\{u>-\infty\}}(v-P(u, v))=0$.

Lemma 6.7. For $u_{0}, u_{1} \in \mathcal{E}^{2}(X, \omega)$ if $d\left(u_{0}, u_{1}\right)=0$ then $u_{0}=u_{1}$.

Proof. Suppose $u_{0}^{k}, u_{1}^{k} \in \mathcal{H}$ are strictly decreasing approximating sequences of $u_{0}, u_{1}$. We also fix $\varepsilon>0$. Let $(0,1) \ni t \rightarrow u_{t}^{k} \in \mathcal{H}_{\Delta}$ be the weak geodesic segment joining $u_{0}^{k}, u_{1}^{k}$. Starting from (15), we have the following sequence of estimates:

$$
\begin{aligned}
d\left(u_{0}^{k}, u_{1}^{k}\right)^{2} & =\int_{X}{\dot{u_{0}^{k}}}^{2}\left(\omega+i \partial \bar{\partial} u_{0}^{k}\right)^{n} \geq \varepsilon^{2} \int_{\left\{\dot{u}_{0}^{k} \geq \varepsilon\right\}}\left(\omega+i \partial \bar{\partial} u_{0}^{k}\right)^{n} \\
& =\varepsilon^{2} \int_{\left\{P\left(u_{0}^{k}, u_{1}^{k}-\varepsilon\right)=u_{0}^{k}\right\}}\left(\omega+i \partial \bar{\partial} u_{0}^{k}\right)^{n}
\end{aligned}
$$

where in the last line we have used Lemma 6.5. As $d\left(u_{0}^{k}, u_{1}^{k}\right) \rightarrow 0$ it follows that

$$
\int_{\left\{P\left(u_{0}^{k}, u_{1}^{k}-\varepsilon\right)=u_{0}^{k}\right\}}\left(\omega+i \partial \bar{\partial} u_{0}^{k}\right)^{n} \rightarrow 0
$$

as $k \rightarrow \infty$. Suppose $\phi \in C^{\infty}(X)$ with $\phi \geq 0$. By Proposition 2.2 we have:

$$
\begin{aligned}
\int_{X} \phi\left(\omega+i \partial \bar{\partial} P\left(u_{0}^{k}, u_{1}^{k}-\varepsilon\right)\right)^{n} \leq \\
\quad \int_{\left\{P\left(u_{0}^{k}, u_{1}^{k}-\varepsilon\right)=u_{0}^{k}\right\}} \phi\left(\omega+i \partial \bar{\partial} u_{0}^{k}\right)^{n}+\int_{\left\{P\left(u_{0}^{k}, u_{1}^{k}-\varepsilon\right)=u_{1}^{k}-\varepsilon\right\}} \phi\left(\omega+i \partial \bar{\partial} u_{1}^{k}\right)^{n},
\end{aligned}
$$

$k \in \mathbb{N}$. Using (37) it results that

$$
\lim _{k \rightarrow \infty} \int_{X} \phi\left(\omega+i \partial \bar{\partial} P\left(u_{0}^{k}, u_{1}^{k}-\varepsilon\right)\right)^{n} \leq \lim _{k \rightarrow \infty} \int_{X} \phi\left(\omega+i \partial \bar{\partial} u_{1}^{k}\right)^{n} .
$$


As $P\left(u_{0}, u_{1}-\varepsilon\right) \in \mathcal{E}^{2}(X, \omega)$ and $P\left(u_{0}^{k}, u_{1}^{k}-\varepsilon\right) \searrow P\left(u_{0}, u_{1}-\varepsilon\right)$, we can conclude:

$$
\left(\omega+i \partial \bar{\partial} P\left(u_{0}, u_{1}-\varepsilon\right)\right)^{n} \leq\left(\omega+i \partial \bar{\partial} u_{1}\right)^{n} .
$$

As in the proof of Theorem 4.3, we have equality in this last estimate, since both measures integrate to $\operatorname{Vol}(X)$. Now S. Dinew's uniqueness theorem implies that

$$
P\left(u_{0}, u_{1}-\varepsilon\right)=u_{1}-\varepsilon-b_{\varepsilon}
$$

for some $b_{\varepsilon} \geq 0$. We will conclude soon that in fact $b_{\varepsilon}=0$, but for the moment let us observe that we can similarly deduce that

$$
P\left(u_{0}-\varepsilon, u_{1}\right)=u_{0}-\varepsilon-c_{\varepsilon}
$$

for some $c_{\varepsilon} \geq 0$. It follows from (38) and (39) that $u_{0}$ and $u_{1}$ have the same singularity type. Lemma 6.6 applied to (38) implies now that either $u_{0}=P\left(u_{0}, u_{1}-\varepsilon\right)=u_{1}-\varepsilon-b_{\varepsilon}$ or $\inf _{X \backslash\left\{u_{0}=-\infty\right\}}\left(u_{1}-\varepsilon-P\left(u_{0}, u_{1}-\varepsilon\right)\right)=0$. The first case implies that $d\left(u_{0}, u_{1}\right)=\varepsilon+b_{\varepsilon}>0$, contradicting our assumption. Hence we have $\inf _{X \backslash\left\{u_{0}=-\infty\right\}}\left(u_{1}-\varepsilon-P\left(u_{0}, u_{1}-\varepsilon\right)\right)=0$ and this implies that $b_{\varepsilon}=0$. We can similarly conclude that $c_{\varepsilon}=0$.

Summing up, we have proved that $P\left(u_{0}, u_{1}-\varepsilon\right)=u_{1}-\varepsilon$ and $P\left(u_{0}-\varepsilon, u_{1}\right)=u_{0}-\varepsilon$. This implies that $u_{1} \geq u_{0}-\varepsilon$ and $u_{0} \geq u_{1}-\varepsilon$. As $\varepsilon>0$ can be chosen arbitrarily small, we conclude that $u_{0}=u_{1}$.

By Corollary 5.4 it follows that given $u_{0}, u_{1} \in \mathcal{E}^{2}(X, \omega)$, for the weak geodesic segment $t \rightarrow u_{t}$ connecting $u_{0}, u_{1}$ we have $u_{t} \in \mathcal{E}^{2}(X, \omega), t \in(0,1)$. Next we show that this weak geodesic is an actual geodesic segment in $\left(\mathcal{E}^{2}(X, \omega), \tilde{d}\right)$ in the sense of (5). Before we do this we need a technical lemma:

Lemma 6.8. Suppose $v_{0}, v_{1} \in \mathcal{H}_{0}$ and $\left\{v_{1}^{j}\right\}_{j \in \mathbb{N}} \subset \mathcal{H}_{0}$ is sequence decreasing to $v_{1}$. By $(0,1) \ni t \rightarrow v_{t}, v_{t}^{j} \in \mathcal{H}_{0}$ we denote the bounded weak geodesic segments connecting $v_{0}, v_{1}$ and $v_{0}, v_{1}^{j}$ respectively. As we have convexity in the $t$-variable, we can define $\dot{v}_{0}=\lim _{t \rightarrow 0}\left(v_{t}-v_{0}\right) / t$ and $\dot{v}_{0}^{j}=\lim _{t \rightarrow 0}\left(v_{t}^{j}-v_{0}\right) / t$. The following holds:

$$
\lim _{j \rightarrow \infty} \int_{X}{\dot{v_{0}^{j}}}^{2}\left(\omega+i \partial \bar{\partial} v_{0}\right)^{n}=\int_{X}{\dot{v_{0}}}^{2}\left(\omega+i \partial \bar{\partial} v_{0}\right)^{n} .
$$

Proof. By (18) there exists $C>0$ such that $\left\|\dot{v}_{0}\right\|_{L^{\infty}(X)},\left\|\dot{v}_{0}^{j}\right\|_{L^{\infty}(X)} \leq C$. We also have $v \leq v^{j}, j \in \mathbb{N}$ by the comparison principle. As we have convexity in the $t$-variable and all our weak geodesics share the same starting point, it also follows that $\dot{v}_{0}^{j} \searrow$ $\dot{v}_{0}$ pointwise. The conclusion of the lemma follows now from Lebesgue's dominated convergence theorem.

Lemma 6.9. Suppose $u_{0}, u_{1} \in \mathcal{E}^{2}(X, \omega)$ and $(0,1) \ni t \rightarrow u_{t} \in \mathcal{E}^{2}(X, \omega)$ is the weak geodesic segment connecting $u_{0}, u_{1}$ defined in (31). Then $t \rightarrow u_{t}$ is a geodesic segment in $\left(\mathcal{E}^{2}(X, \omega), \tilde{d}\right)$ in the sense of (5)).

Proof. We have $u_{t} \in \mathcal{E}^{2}(X, \omega), t \in[0,1]$, as follows from Corollarly 5.4. First we prove that

$$
\tilde{d}\left(u_{0}, u_{l}\right)=l \tilde{d}\left(u_{0}, u_{1}\right)
$$


for $l \in[0,1]$. Suppose $u_{0}^{k}, u_{1}^{k} \in \mathcal{H}$ are strictly decreasing approximating sequences of $u_{0}, u_{1}$ and let $(0,1) \ni t \rightarrow u_{t}^{k} \in \mathcal{H}_{\Delta}$ be the decreasing sequence of weak geodesics connecting $u_{0}^{k}, u_{1}^{k}$. By definition, we have that

$$
\tilde{d}\left(u_{0}, u_{1}\right)=\lim _{k \rightarrow \infty} d\left(u_{0}^{k}, u_{1}^{k}\right)=\lim _{k \rightarrow \infty} \sqrt{\int_{X}{\dot{u_{0}^{k}}}^{2}\left(\omega+i \partial \bar{\partial} u_{0}^{k}\right)^{n}}
$$

where we have used (15).

The geodesics segments $(0,1) \ni t \rightarrow u_{t}^{k} \in \mathcal{H}_{\Delta}$ are decreasing pointwise to $(0,1) \ni$ $t \rightarrow u_{t} \in \mathcal{E}^{2}(X, \omega)$. In particular, this implies that $u_{l}^{k} \searrow u_{l}$. We want to find a decreasing sequence $v_{l}^{k} \in \mathcal{H}$ such that $u_{l}^{k} \leq v_{l}^{k}, v_{l}^{k} \searrow u_{l}$ and

$$
l d\left(u_{0}^{k}, u_{1}^{k}\right)-d\left(u_{0}^{k}, v_{l}^{k}\right)=l \sqrt{\int_{X} \dot{u}_{0}^{2}\left(\omega+i \partial \bar{\partial} u_{0}^{k}\right)^{n}}-\sqrt{\int_{X}{\dot{v_{0}^{k}}}^{2}\left(\omega+i \partial \bar{\partial} u_{0}^{k}\right)^{n}} \rightarrow 0
$$

as $k \rightarrow \infty$, where $(0,1) \ni t \rightarrow v^{k} \in \mathcal{H}_{\Delta}$ is the weak geodesic segment connecting $u_{0}^{k}$ and $v_{l}^{k}$. By the definition of $\tilde{d}$, letting $k \rightarrow \infty$ in (41) would give us (40).

Finding such sequence $v_{l}^{k}$ is always possible by an application of the previous lemma to $v_{0}:=u_{0}^{k}$ and $v_{1}:=u_{l}^{k}$, as we observe that the weak geodesic segment connecting $u_{0}^{k}$ and $u_{l}^{k}$ is exactly $t \rightarrow u_{l t}^{k}$.

Finally, we prove that for $t_{1}, t_{2} \in[0,1], t_{1} \leq t_{2}$ we have

$$
\tilde{d}\left(u_{t_{1}}, u_{t_{2}}\right)=\left(t_{2}-t_{1}\right) \tilde{d}\left(u_{0}, u_{1}\right) .
$$

Let $h_{0}=u_{t_{2}}$ and $h_{1}=u_{0}$. If $(0,1) \ni t \rightarrow h_{t} \in \mathcal{E}^{2}(X, \omega)$ is the weak geodesic connecting $h_{0}, h_{1}$ as defined in (31), using (32) one can easily see that $h_{t}=u_{t_{2}(1-t)}$. Applying (40) to $t \rightarrow h_{t}$ and $l=1-t_{1} / t_{2}$ we obtain

$$
\left(1-t_{1} / t_{2}\right) \tilde{d}\left(u_{t_{2}}, u_{0}\right)=\tilde{d}\left(u_{t_{2}}, u_{t_{1}}\right) .
$$

Now applying (40) for $t \rightarrow u_{t}$ and $l=t_{2}$ we have

$$
\tilde{d}\left(u_{0}, u_{t_{2}}\right)=t_{2} \tilde{d}\left(u_{0}, u_{1}\right) .
$$

Putting these last two formulas together we obtain (42), finishing the proof.

The following one sided generalization of Lemma 6.2 will be very useful:

Lemma 6.10. Suppose $u, v \in \mathcal{E}^{2}(X, \omega)$ satisfying $u \leq v$. Then:

$$
\tilde{d}(u, v)^{2} \leq C \int_{X}(v-u)^{2}(\omega+i \partial \bar{\partial} u)^{n},
$$

where $C>0$ only depends on $\operatorname{dim} X$.

Proof. Let $u_{k}, v_{k} \in \mathcal{H}$ be sequences decreasing to $u, v$, with the additional property $u_{k} \leq v_{k}, k \geq 1$. By Lemma 6.2 we have:

$$
d\left(u_{k}, v_{k}\right)^{2} \leq \int_{X}\left(u_{k}-v_{k}\right)^{2}\left(\omega+i \partial \bar{\partial} u_{k}\right)^{n} .
$$


We clearly have $u-v_{k}, u_{k}-v_{k} \in \mathcal{E}^{2}\left(X, \omega+i \partial \bar{\partial} v_{k}\right)$ and $u-v_{k} \leq u_{k}-v_{k} \leq 0$. Applying Proposition 2.5 to $\mathcal{E}^{2}\left(X, \omega+i \partial \bar{\partial} v_{k}\right)$ we can conclude:

$$
d\left(u_{k}, v_{k}\right)^{2} \leq C \int_{X}\left(u-v_{k}\right)^{2}(\omega+i \partial \bar{\partial} u)^{n}
$$

Letting $k \rightarrow \infty$, by the dominated convergence theorem we arrive at the desired estimate.

We prove now that monotone sequences in $\mathcal{E}^{2}(X, \omega)$ converge with respect to the Mabuchi metric:

Proposition 6.11. If $\left\{w_{k}\right\}_{k \in \mathbb{N}} \subset \mathcal{E}^{2}(X, \omega)$ decreases (increases a.e.) to $w \in \mathcal{E}^{2}(X, \omega)$ then $\tilde{d}\left(w_{k}, w\right) \rightarrow 0$.

Proof. When $w_{k}$ is decreasing to $w$ the result follows from the estimate we just proved and the dominated convergence theorem. Suppose $w_{k}$ increases a.e. to $w$. Again, using the previous Lemma we want to prove that

$$
\int_{X}\left(w-w_{k}\right)^{2}\left(\omega+i \partial \bar{\partial} w_{k}\right)^{n} \rightarrow 0
$$

The rest of the argument is adapted from [BEGZ, Theorem 2.17]. We can additionally suppose that all the functions are negative. Let $w_{k}^{L}=\max \left\{w_{k},-L\right\}, L \geq 0$ with $w^{L}$ defined similarly. If the $w_{k}$ are uniformly bounded then (43) can be seen using the quasicontinuity property of pluri-subharmonic functions. Hence we are done if we can prove that the quantity

$$
\left|\int_{X}\left(w-w_{k}\right)^{2}\left(\omega+i \partial \bar{\partial} w_{k}\right)^{n}-\int_{X}\left(w^{L}-w_{k}^{L}\right)^{2}\left(\omega+i \partial \bar{\partial} w_{k}^{L}\right)^{n}\right|
$$

tends to zero uniformly as $L \rightarrow \infty$. Before we get into the estimates we observe that there exists $\chi \in \mathcal{W}_{M}^{+}$for some $M>1$ such that $-t^{2} / \chi(t)$ decreases to 0 as $t \rightarrow-\infty$ and $w_{k}, w \in \mathcal{E}_{\chi}(X, \omega), k \in \mathbb{N}$. We can write:

$$
\begin{aligned}
\mid \int_{X}\left(w-w_{k}\right)^{2} & \left(\omega+i \partial \bar{\partial} w_{k}\right)^{n}-\int_{X}\left(w^{L}-w_{k}^{L}\right)^{2}\left(\omega+i \partial \bar{\partial} w_{k}^{L}\right)^{n} \mid \leq \\
\leq & \left|\int_{\left\{w_{k} \leq-L\right\}}\left(w-w_{k}\right)^{2}\left(\omega+i \partial \bar{\partial} w_{k}\right)^{n}-\int_{\left\{w_{k} \leq-L\right\}}\left(w^{L}-w_{k}^{L}\right)^{2}\left(\omega+i \partial \bar{\partial} w_{k}^{L}\right)^{n}\right| \\
& \leq\left(\int_{\left\{w_{k} \leq-L\right\}} w_{k}^{2}\left(\omega+i \partial \bar{\partial} w_{k}\right)^{n}+\int_{\left\{w_{k} \leq-L\right\}} w_{k}^{L^{2}}\left(\omega+i \partial \bar{\partial} w_{k}^{L}\right)^{n}\right) \\
& \leq \frac{L^{2}}{\chi(L)}\left(\int_{\left\{w_{k} \leq-L\right\}} \chi\left(w_{k}\right)\left(\omega+i \partial \bar{\partial} w_{k}\right)^{n}+\int_{\left\{w_{k} \leq-L\right\}} \chi\left(w_{k}^{L}\right)\left(\omega+i \partial \bar{\partial} w_{k}^{L}\right)^{n}\right) \\
& \leq \frac{C L^{2}}{|\chi(L)|}\left|E_{\chi}\left(w_{k}\right)\right| \leq \frac{C L^{2}}{\chi(L)},
\end{aligned}
$$

where in the last line we have used Proposition 2.5 ,

Lemma 6.12. $\left(\mathcal{E}^{2}(X, \omega), \tilde{d}\right)$ is non-positively curved in the sense of Alexandrov. In particular, goedesic segments joining different points of $\mathcal{E}^{2}(X, \omega)$ are unique. 
Proof. Suppose $p, q, r \in \mathcal{E}^{2}(X, \omega)$ and $(0,1) \ni t \rightarrow u_{t}^{q r} \in \mathcal{E}^{2}(X, \omega)$ is the weak geodesic segment connecting $q, r$. Suppose $s \in\left\{u^{q r}\right\}$ with $\lambda \tilde{d}(q, r)=\tilde{d}(q, s)$, i.e. $s=u_{\lambda}^{q r}$. We need to prove the estimate

$$
\tilde{d}(p, s)^{2} \leq \lambda \tilde{d}(p, r)^{2}+(1-\lambda) \tilde{d}(p, q)^{2}-\lambda(1-\lambda) \tilde{d}(q, r)^{2} .
$$

First, suppose $p, q, r \in \mathcal{H}$. The proof of the above inequality in this case is done in [CC], so we refer to this work and only skim over the argument.

We fix $\varepsilon, \varepsilon^{\prime}>0$. With $[0,1] \ni t \rightarrow u_{t}^{\varepsilon, p q}, u_{t}^{\varepsilon, p r}, u_{t}^{\varepsilon^{\prime}, q r} \in \mathcal{H}$ we denote the smooth $\varepsilon$-geodesics $\left(\varepsilon^{\prime}\right.$-geodesics) joining $(p, q),(p, r)$ and $(q, r)$ respectively (see Section 2.1). Given a smooth curve $[0,1] \ni t \rightarrow \alpha_{t} \in \mathcal{H}$, we denote by $L(\alpha)$ its energy:

$$
L(\alpha)=\int_{0}^{1} \int_{X} \dot{\alpha}_{t}^{2}\left(\omega+i \partial \bar{\partial} \alpha_{t}\right)^{n} .
$$

Let $[0,1] \ni t \rightarrow u_{t}^{\varepsilon, \lambda} \in \mathcal{H}$ be the $\varepsilon$-geodesic joining $p$ and $u_{\lambda}^{\varepsilon^{\prime}, q r}$. It is proved in [CC, Section 2.4] that

$$
L\left(u^{\varepsilon, \lambda}\right) \leq(1-\lambda) L\left(u^{\varepsilon, p q}\right)+\lambda L\left(u^{\varepsilon, p r}\right)-\lambda(1-\lambda) L\left(u^{\varepsilon^{\prime}, q r}\right)-\left(\varepsilon+\varepsilon^{\prime}\right) C
$$

where $C>0$ is independent of $\varepsilon, \varepsilon^{\prime}$. Using the same ideas that lead to (15), after letting first $\varepsilon \rightarrow 0$ then $\varepsilon^{\prime} \rightarrow 0$ in the above estimate we arrive at

$$
\tilde{d}(p, s)^{2}=\tilde{d}\left(p, u_{\lambda}\right)^{2} \leq(1-\lambda) \tilde{d}(p, q)^{2}+\lambda \tilde{d}(p, r)^{2}-\lambda(1-\lambda) \tilde{d}(q, r)^{2} .
$$

In general, if $p, q, r \in \mathcal{E}^{2}(X, \omega)$, we can use approximation: suppose $p_{k}, q_{k}, r_{k} \in \mathcal{H}$ are sequences decreasing to $p, q, r$. From what we proved it follows that

$$
\tilde{d}\left(p^{k}, u_{\lambda}^{q^{k} r^{k}}\right)^{2} \leq(1-\lambda) \tilde{d}\left(p^{k}, q^{k}\right)^{2}+\lambda \tilde{d}\left(p^{k}, r^{k}\right)^{2}-\lambda(1-\lambda) \tilde{d}\left(q^{k}, r^{k}\right)^{2}
$$

where $(0,1) \ni t \rightarrow u_{t}^{q^{k} r^{k}} \in \mathcal{H}_{\Delta}$ is the weak geodesic connecting $q^{k}, r^{k}$. From the comparison principle it follows that $\left\{u^{q^{k} r^{k}}\right\}_{k}$ is decreasing to $u^{q r}$, in particular $u_{\lambda}^{q^{k} r^{k}} \searrow u_{\lambda}^{q r}=s$. Using Proposition 6.11 we can take the limit in this last estimate to obtain (44).

As a corollary to Theorem 6.1, using the estimates of Berndtsson (18), Blocki Bl3, Theorem 1], He [프, Theorem 1.1] and Theorem [5, we obtain that $\left(\mathcal{E}^{2}(X, \omega), \tilde{d}\right)$ has many special dense totally geodesic subspaces:

Corollary 6.13. $\mathcal{H}$ is dense in $\mathcal{E}^{2}(X, \omega)$ and the following sets are totally geodesic dense subspaces of $\left(\mathcal{E}^{2}(X, \omega), \tilde{d}\right)$ :

(i) $\mathcal{H}_{\Delta}=\left\{u \in \operatorname{PSH}(X, \omega) \mid \Delta u \in L^{\infty}(X)\right\}$;

(ii) $\mathcal{H}_{0,1}=\operatorname{PSH}(X, \omega) \cap \operatorname{Lip}(X)$;

(iii) $\mathcal{H}_{0}=\operatorname{PSH}(X, \omega) \cap L^{\infty}(X)$;

(iv) $\mathcal{E}_{\chi}(X, \omega)$, if $\chi \in \mathcal{W}_{M}^{+}, M \geq 1$ satisfies $\lim \sup _{t \rightarrow-\infty} \chi(t) / t^{2} \leq-1$.

Proof. Suppose $\mathcal{S}$ is one of the above subspaces. From the above quoted results it follows that given $u_{0}, u_{1} \in \mathcal{S}$, for the weak geodesic segment $t \rightarrow u_{t}$ joining $u_{0}, u_{1}$ we have $u_{t} \in \mathcal{S}, t \in(0,1)$.

As $\mathcal{H} \subset \mathcal{S}$, we just need to argue that $\mathcal{H} \subset \mathcal{E}^{2}(X, \omega)$ is dense. As elements of $\mathcal{E}^{2}(X, \omega)$ can be approximated by decreasing sequences in $\mathcal{H}$, an application of Proposition 6.11 finishes the proof. 


\section{The Length of Geodesic Segments}

In light of results obtained in the previous section, one would like to generalize formula (15) for arbitrary $u_{0}, u_{1} \in \mathcal{E}^{2}(X, \omega)$. However this fails even for $u_{0}, u_{1} \in \mathcal{H}_{0,1}$, as the next (familiar) example shows. Suppose $\operatorname{dim} X=1$ and $g_{x} \in \operatorname{PSH}(X, \omega)$ is the $\omega$-Green function with pole at some $x \in X$. As in Section 2.2, let $u_{0}=\max \left(g_{x}, 0\right)$ and $u_{1}=0$. We denote by $(0,1) \ni t \rightarrow u_{t} \in \mathcal{H}_{0}$ the geodesic connecting $u_{0}$ and $u_{1}$. Clearly $\tilde{d}\left(u_{0}, u_{1}\right)>0$. By properties of Green functions, $\left(\omega+i \partial \bar{\partial} u_{0}\right)$ only charges the set $\left\{g_{x} \leq 0\right\}$. However $\left.\dot{u}_{0}\right|_{\left\{g_{x} \leq 0\right\}} \equiv 0$, because $0 \leq u_{t}, t \in[0,1]$ and $t \rightarrow u_{t}$ is decreasing. It results that

$$
\int_{X} \dot{u}_{0}^{2}\left(\omega+i \partial \bar{\partial} u_{0}\right)=0
$$

contradicting (15). This example also suggests that one can not endow $\mathcal{E}^{2}(X, \omega)$ with a Riemannian structure that would induce the metric space $\left(\mathcal{E}^{2}(X, \omega), \tilde{d}\right)$ unlike $(\mathcal{H}, d)$.

The main purpose of this section is to show that (15) nevertheless holds for $u_{0}, u_{1} \in$ $\mathcal{H}_{\Delta}$. Recall that by [BD, Corollary 4.7] (see also [H, Theorem 1.1]) we have $u_{t} \in \mathcal{H}_{\Delta}, t \in$ $(0,1)$, where $t \rightarrow u_{t}$ is the geodesic joining $u_{0}, u_{1}$. We start with a lemma:

Lemma 7.1. Suppose $u_{0}, u_{1} \in \mathcal{H}_{\Delta}$ and $(0,1) \ni t \rightarrow u_{t} \in \mathcal{H}_{\Delta}$ is the geodesic connecting them. Then the following holds:

$$
\int_{X} \dot{u}_{0}^{2}\left(\omega+i \partial \bar{\partial} u_{0}\right)^{n}=\int_{X} \dot{u}_{1}^{2}\left(\omega+i \partial \bar{\partial} u_{1}\right)^{n} .
$$

Proof. To obtain (45) we prove the following two formulas:

$$
\begin{aligned}
\int_{\left\{\dot{u}_{0}>0\right\}} \dot{u}_{0}^{2}\left(\omega+i \partial \bar{\partial} u_{0}\right)^{n} & =\int_{\left\{\dot{u}_{1}>0\right\}} \dot{u}_{1}^{2}\left(\omega+i \partial \bar{\partial} u_{1}\right)^{n}, \\
\int_{\left\{\dot{u}_{0}<0\right\}} \dot{u}_{0}^{2}\left(\omega+i \partial \bar{\partial} u_{0}\right)^{n} & =\int_{\left\{\dot{u}_{1}<0\right\}} \dot{u}_{1}^{2}\left(\omega+i \partial \bar{\partial} u_{1}\right)^{n} .
\end{aligned}
$$

Using Remark 2.3 and Lemma 6.5 multiple times we can write:

$$
\begin{aligned}
\int_{\left\{\dot{u}_{0}>0\right\}} \dot{u}_{0}^{2}\left(\omega+i \partial \bar{\partial} u_{0}\right)^{n} & =2 \int_{0}^{\infty} \tau\left(\omega+i \partial \bar{\partial} u_{0}\right)^{n}\left(\left\{\dot{u}_{0} \geq \tau\right\}\right) d \tau \\
& =2 \int_{0}^{\infty} \tau\left(\omega+i \partial \bar{\partial} u_{0}\right)^{n}\left(\left\{P\left(u_{0}, u_{1}-\tau\right)=u_{0}\right\}\right) d \tau \\
& =2 \int_{0}^{\infty} \tau\left(\operatorname{Vol}(X)-\left(\omega+i \partial \bar{\partial} u_{1}\right)^{n}\left(\left\{P\left(u_{0}, u_{1}-\tau\right)=u_{1}-\tau\right\}\right)\right) d \tau \\
& =2 \int_{0}^{\infty} \tau\left(\omega+i \partial \bar{\partial} u_{1}\right)^{n}\left(\left\{P\left(u_{0}, u_{1}-\tau\right)<u_{1}-\tau\right\}\right) d \tau \\
& =2 \int_{0}^{\infty} \tau\left(\omega+i \partial \bar{\partial} u_{1}\right)^{n}\left(\left\{P\left(u_{0}+\tau, u_{1}\right)<u_{1}\right\}\right) d \tau \\
& =2 \int_{0}^{\infty} \tau\left(\omega+i \partial \bar{\partial} u_{1}\right)^{n}\left(\left\{\dot{u}_{1}>\tau\right\}\right) d \tau \\
& =\int_{\left\{\dot{u}_{1}>0\right\}} \dot{u}_{1}^{2}\left(\omega+i \partial \bar{\partial} u_{1}\right)^{n}
\end{aligned}
$$

where in the second we have used Lemma 6.5, in the third line we have used Remark 2.3 and in the sixth line we have used Lemma 6.5 again. Formula (47) follows if we apply (46) to the "reversed" geodesic $t \rightarrow v_{t}=u_{1-t}$. 
Theorem 7.2. Suppose $u_{0}, u_{1} \in \mathcal{H}_{\Delta}$ and $(0,1) \ni t \rightarrow u_{t} \in \mathcal{H}_{\Delta}$ is the geodesic connecting them. Then we have:

$$
\tilde{d}\left(u_{0}, u_{1}\right)^{2}=\int_{X} \dot{u}_{t}^{2}\left(\omega+i \partial \bar{\partial} u_{t}\right)^{n}, t \in[0,1] .
$$

Proof. As usual, let $u_{0}^{k}, u_{1}^{k} \in \mathcal{H}$ be a sequence of potentials decreasing to $u_{0}, u_{1}$. Let $(0,1) \ni t \rightarrow u_{t}^{k l} \in \mathcal{H}_{\Delta}$ be the geodesic joining $u_{0}^{k}, u_{1}^{l}$. By (15) we have

$$
\tilde{d}\left(u_{0}^{k}, u_{1}^{l}\right)^{2}=\int_{X} \dot{u_{0}^{k l}}{ }^{2}\left(\omega+i \partial \bar{\partial} u_{0}^{k}\right)^{n} .
$$

If we let $l \rightarrow \infty$, by Lemma 6.8 and Proposition 6.11 we obtain that that

$$
\tilde{d}\left(u_{0}^{k}, u_{1}\right)^{2}=\int_{X}{\dot{u_{0}^{k}}}^{2}\left(\omega+i \partial \bar{\partial} u_{0}^{k}\right)^{n}
$$

where $(0,1) \ni t \rightarrow u_{t}^{k} \in \mathcal{H}_{\Delta}$ is the geodesic connecting $u_{0}^{k}$ with $u_{1}$. Using the previous lemma we can write:

$$
\tilde{d}\left(u_{0}^{k}, u_{1}\right)^{2}=\int_{X}{\dot{u_{1}^{k}}}^{2}\left(\omega+i \partial \bar{\partial} u_{1}\right)^{n} .
$$

Letting $k \rightarrow \infty$, another application of Lemma 6.8 yields (48) for $t=1$. The case $t=0$ follows by symmetry, and for $0<t<1$ the result follows because a subarc of a geodesic is again a geodesic.

\section{$8 \quad$ Metric Properties of the Operator $(u, v) \rightarrow P(u, v)$}

In this short section we explore the geometry of the operator $(u, v) \rightarrow P(u, v)$ restricted to the space $\mathcal{E}^{2}(X, \omega)$. First we observe that the triplet $(u, v, P(u, v))$ always forms a right triangle. This will help in proving that $P(\cdot, \cdot)$ contracts distances with respect to $\tilde{d}$ in both components.

Proposition 8.1 (Pythagorean formula). Given $u_{0}, u_{1} \in \mathcal{E}^{2}(X, \omega)$, we have $P\left(u_{0}, u_{1}\right) \in$ $\mathcal{E}^{2}(X, \omega)$ and

$$
\tilde{d}\left(u_{0}, u_{1}\right)^{2}=\tilde{d}\left(u_{0}, P\left(u_{0}, u_{1}\right)\right)^{2}+\tilde{d}\left(P\left(u_{0}, u_{1}\right), u_{1}\right)^{2} .
$$

Proof. By Proposition 6.11, it is enough to prove the above formula for $u_{0}, u_{1} \in \mathcal{H}$. According to Theorem 2.1 we have $P\left(u_{0}, u_{1}\right) \in \mathcal{H}_{\Delta}$. Suppose $(0,1) \ni t \rightarrow u_{t} \in \mathcal{H}_{\Delta}$ is the geodesic connecting $u_{0}, u_{1}$. By (15):

$$
d\left(u_{0}, u_{1}\right)^{2}=\int_{X} \dot{u}_{0}^{2}\left(\omega+i \partial \bar{\partial} u_{0}\right)^{n}
$$

To complete the argument we will prove the following:

$$
\begin{aligned}
& d\left(u_{1}, P\left(u_{0}, u_{1}\right)\right)^{2}=\int_{\left\{\dot{u}_{0}>0\right\}} \dot{u}_{0}^{2}\left(\omega+i \partial \bar{\partial} u_{0}\right)^{n} \\
& d\left(u_{0}, P\left(u_{0}, u_{1}\right)\right)^{2}=\int_{\left\{\dot{u}_{0}<0\right\}} \dot{u}_{0}^{2}\left(\omega+i \partial \bar{\partial} u_{0}\right)^{n} .
\end{aligned}
$$


We prove now (49). Using Lemma 6.5 we can write:

$$
\begin{aligned}
\int_{\left\{\dot{u}_{0}>0\right\}} \dot{u}_{0}^{2}\left(\omega+i \partial \bar{\partial} u_{0}\right)^{n} & =2 \int_{0}^{\infty} \tau\left(\omega+i \partial \bar{\partial} u_{0}\right)^{n}\left(\left\{\dot{u}_{0} \geq \tau\right\}\right) d \tau \\
& =2 \int_{0}^{\infty} \tau\left(\omega+i \partial \bar{\partial} u_{0}\right)^{n}\left(\left\{P\left(u_{0}, u_{1}-\tau\right)=u_{0}\right\}\right) d \tau
\end{aligned}
$$

Suppose $(0,1) \ni t \rightarrow \tilde{u}_{t} \in \mathcal{H}_{\Delta}$ is the weak geodesic connecting $P\left(u_{0}, u_{1}\right), u_{1}$. As $(t, x) \rightarrow$ $\tilde{u}_{t}(x)$ is increasing in the $t$-variable, we have $\dot{\tilde{u}}_{0} \geq 0$. By Theorem [7.2, Lemma 6.5 and Proposition 2.2 we can write

$$
\begin{aligned}
\tilde{d}\left(P\left(u_{0}, u_{1}\right), u_{1}\right)^{2} & =\int_{X} \dot{\tilde{u}}_{0}^{2}\left(\omega+i \partial \bar{\partial} P\left(u_{0}, u_{1}\right)\right)^{n}=\int_{\left\{\dot{\tilde{u}}_{0}>0\right\}} \dot{\tilde{u}}_{0}^{2}\left(\omega+i \partial \bar{\partial} P\left(u_{0}, u_{1}\right)\right)^{n} \\
& =2 \int_{0}^{\infty} \tau\left(\omega+i \partial \bar{\partial} P\left(u_{0}, u_{1}\right)\right)^{n}\left(\left\{\dot{\tilde{u}}_{0} \geq \tau\right\}\right) d \tau \\
& =2 \int_{0}^{\infty} \tau\left(\omega+i \partial \bar{\partial} P\left(u_{0}, u_{1}\right)\right)^{n}\left(\left\{P\left(P\left(u_{0}, u_{1}\right), u_{1}-\tau\right)=P\left(u_{0}, u_{1}\right)\right\}\right) d \tau \\
& =2 \int_{0}^{\infty} \tau\left(\omega+i \partial \bar{\partial} P\left(u_{0}, u_{1}\right)\right)^{n}\left(\left\{P\left(u_{0}, u_{1}-\tau\right)=P\left(u_{0}, u_{1}\right)\right\}\right) d \tau \\
& =2 \int_{0}^{\infty} \tau\left(\omega+i \partial \bar{\partial} u_{0}\right)^{n}\left(\left\{P\left(u_{0}, u_{1}-\tau\right)=P\left(u_{0}, u_{1}\right)=u_{0}\right\}\right) d \tau \\
& =2 \int_{0}^{\infty} \tau\left(\omega+i \partial \bar{\partial} u_{0}\right)^{n}\left(\left\{P\left(u_{0}, u_{1}-\tau\right)=u_{0}\right\}\right) d \tau
\end{aligned}
$$

where in the third line we have used Lemma 6.5, in the fifth line we have used Proposition 2.2 and the fact that $\left\{P\left(u_{0}, u_{1}\right)=u_{1}\right\} \cap\left\{P\left(u_{0}, u_{1}-\tau\right)=P\left(u_{0}, u_{1}\right)\right\}$ is empty for $\tau>0$.

From our calculations (49) follows. One can conclude (50) from (49) after reversing the roles of $u_{0}, u_{1}$ and then using (46).

Proposition 8.2. Given $u, v, w \in \mathcal{E}^{2}(X, \omega)$ we have

$$
\tilde{d}(P(u, v), P(u, w)) \leq \tilde{d}(v, w) .
$$

Proof. First we assume that $v \leq w$. As before, we can also assume $u, v, w \in \mathcal{H}$ and $P(u, v), P(u, w) \in \mathcal{H}_{\Delta}$. Let $(0,1) \ni t \rightarrow \phi_{t}, \psi_{t} \in \mathcal{H}_{\Delta}$ be geodesic segments, $\phi_{t}$ connecting $v, w$ and $\psi_{t}$ connecting $P(u, v), P(u, w)$. By Theorem 7.2 we have to argue that

$$
\int_{X} \dot{\psi}_{0}^{2}(\omega+i \partial \bar{\partial} P(u, v))^{n} \leq \int_{X} \dot{\phi}_{0}^{2}(\omega+i \partial \bar{\partial} v)^{n} .
$$

Proposition 2.2 implies that

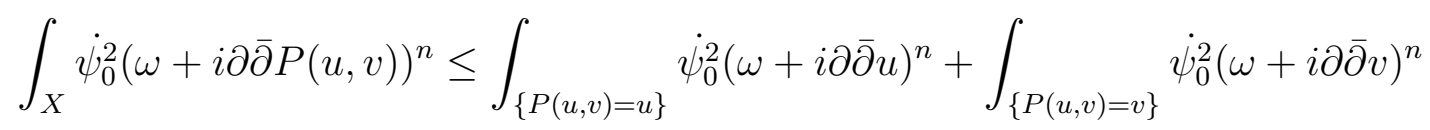

We argue that the first term in this sum is zero. As $P(u, v) \leq P(u, w)$, it is clear that $t \rightarrow \psi_{t}$ is increasing in $t$. By the maximum principle, it is also clear that $\psi_{t} \leq u, t \in[0,1]$. Hence, if $x \in\{P(u, v)=u\}$ then $\psi_{t}(x)=u(x), t \in[0,1]$, implying $\left.\dot{\psi}_{0}\right|_{\{P(u, v)=u\}} \equiv 0$.

At the same time, using the maximum principle again, it follows that $\psi_{t} \leq \phi_{t}, t \in$ $[0,1]$. This implies that $0 \leq\left.\dot{\psi}_{0}\right|_{\{P(u, v)=v\}} \leq\left.\dot{\phi}_{0}\right|_{\{P(u, v)=v\}}$, which in turn implies (51). 
The general case follows now from an application of the Pythagorean formula (Proposition 8.1) and what we just proved:

$$
\begin{aligned}
\tilde{d}(P(u, v), P(u, w))^{2} & =\tilde{d}(P(u, v), P(u, v, w))^{2}+d(P(u, w), P(u, v, w))^{2} \\
& =\tilde{d}(P(u, v), P(u, P(v, w)))^{2}+d(P(u, w), P(u, P(v, w)))^{2} \\
& \leq \tilde{d}(v, P(v, w))^{2}+d(w, P(v, w))^{2} \\
& =\tilde{d}(v, w)^{2} .
\end{aligned}
$$

\section{Completeness of $\left(\mathcal{E}^{2}(X, \omega), \tilde{d}\right)$}

We recall that the Aubin-Mabuchi energy is a functional $A M: \mathcal{H}_{0} \rightarrow \mathbb{R}$ defined by the formula:

$$
A M(v)=\frac{1}{n+1} \sum_{j=0}^{n} \int_{X} v \omega^{j} \wedge(\omega+i \partial \bar{\partial} v)^{n-j} .
$$

As an easy computation shows, for $u, v \in \mathcal{H}_{0}$ we have

$$
A M(u)-A M(v)=\frac{1}{n+1} \sum_{j=0}^{n} \int_{X}(u-v)(\omega+i \partial \bar{\partial} u)^{j} \wedge(\omega+i \partial \bar{\partial} v)^{n-j} .
$$

As the growth of $A M(\cdot)$ is the same as the growth of $E_{\chi^{1}}(\cdot)$ ([BEGZ, Proposition 2.8]), one can extend $A M(\cdot)$ to $\mathcal{E}^{1}(X, \omega)$. We observe now that the Aubin-Mabuchi energy is Lipschitz continuous with respect to the Mabuchi metric:

Lemma 9.1. Given $u_{0}, u_{1} \in \mathcal{E}^{2}(X, \omega)$, we have $\left|A M\left(u_{0}\right)-A M\left(u_{1}\right)\right| \leq \sqrt{\operatorname{Vol}(X)} \tilde{d}\left(u_{0}, u_{1}\right)$. Proof. By density we can suppose that $u_{0}, u_{1} \in \mathcal{H}$. Let $(0,1) \ni t \rightarrow u_{t} \in \mathcal{H}_{\Delta}$ be the geodesic connecting $u_{0}, u_{1}$. By (15), (52) and the Cauchy-Schwarz inequality we have:

$$
\begin{aligned}
A M\left(u_{1}\right)-A M\left(u_{0}\right) & =\int_{0}^{1} \frac{d A M\left(u_{t}\right)}{d t} d t=\int_{0}^{1} \int_{X} \dot{u}_{t}\left(\omega+i \partial \bar{\partial} u_{t}\right)^{n} d t \\
& \leq \int_{0}^{1} \sqrt{\operatorname{Vol}(X)} \sqrt{\int_{X} \dot{u_{t}^{2}}\left(\omega+i \partial \bar{\partial} u_{t}\right)^{n}} d t=\sqrt{\operatorname{Vol}(X)} d\left(u_{0}, u_{1}\right) .
\end{aligned}
$$

We are ready to prove completeness of $\left(\mathcal{E}^{2}(X, \omega), \tilde{d}\right)$. Roughly, the idea of the proof is to replace an arbitrary Cauchy sequence with an equivalent monotone Cauchy sequence which is much easier to deal with in light of Proposition 6.11 and Lemma 9.4,

Theorem 9.2. $\left(\mathcal{E}^{2}(X, \omega), \tilde{d}\right)$ is complete.

Proof. In Corollary 6.13 we have seen that $\mathcal{H}$ is a dense subset of $\mathcal{E}^{2}(X, \omega)$. Suppose $\left\{u_{k}\right\}_{k \in \mathbb{N}} \subset \mathcal{H}$ is a $\tilde{d}$-Cauchy sequence with respect to the Mabuchi metric. We will prove that there exists $v \in \mathcal{E}^{2}(X, \omega)$ such that $\tilde{d}\left(v, u_{k}\right) \rightarrow 0$. After passing to a subsequence we can assume that

$$
\tilde{d}\left(u_{l}, u_{l+1}\right) \leq 1 / 2^{l}, l \in \mathbb{N} .
$$


We introduce $v_{l}^{k}=P\left(u_{k}, u_{k+1}, \ldots, u_{k+l}\right) \in \mathcal{H}_{0}, l, k \in \mathbb{N}$. We argue first that each decreasing sequence $\left\{v_{l}^{k}\right\}_{l \in \mathbb{N}}$ is $\tilde{d}$-Cauchy. Given our assumptions, this fill follow if we show that $\tilde{d}\left(v_{l+1}^{k}, v_{l}^{k}\right) \leq \tilde{d}\left(u_{l+1}, u_{l}\right)$. We observe that $v_{l+1}^{k}=P\left(v_{l}^{k}, u_{k+l+1}\right)$ and $v_{l}^{k}=$ $P\left(v_{l}^{k}, u_{k+l}\right)$. Using this and Proposition 8.2 we can write:

$$
\tilde{d}\left(v_{l+1}^{k}, v_{l}^{k}\right)=\tilde{d}\left(P\left(v_{l}^{k}, u_{k+l+1}\right), P\left(v_{l}^{k}, u_{k+l}\right)\right) \leq \tilde{d}\left(u_{k+l+1}, u_{k+l}\right) \leq \frac{1}{2^{k+l}} .
$$

As we show below in Lemma 9.4, it follows now that each sequence $\left\{v_{l}^{k}\right\}_{l \in \mathbb{N}}$ is $\tilde{d}$-convergening to some $v^{k} \in \mathcal{E}^{2}(X, \omega)$. Using the same trick as above, one can prove:

$$
\begin{aligned}
\tilde{d}\left(v^{k}, v^{k+1}\right)=\lim _{l \rightarrow \infty} \tilde{d}\left(v_{l+1}^{k}\right. & \left., v_{l}^{k+1}\right)=\lim _{l \rightarrow \infty} \tilde{d}\left(P\left(u_{k}, v_{l}^{k+1}\right), P\left(u_{k+1}, v_{l}^{k+1}\right)\right) \leq \tilde{d}\left(u_{k}, u_{k+1}\right) \leq \frac{1}{2^{k}} \\
\tilde{d}\left(v^{k}, u_{k}\right) & =\lim _{l \rightarrow \infty} \tilde{d}\left(v_{l}^{k}, u_{k}\right)=\lim _{l \rightarrow \infty} \tilde{d}\left(\left(P\left(u_{k}, v_{l-1}^{k+1}\right), P\left(u_{k}, u_{k}\right)\right)\right. \\
& \leq \lim _{l \rightarrow \infty} \tilde{d}\left(v_{l-1}^{k+1}, u_{k}\right)=\lim _{l \rightarrow \infty} \tilde{d}\left(P\left(u_{k+1}, v_{l-2}^{k+2}\right), u_{k}\right) \\
& \leq \lim _{l \rightarrow \infty} \tilde{d}\left(P\left(u_{k+1}, v_{l-2}^{k+2}\right), u_{k+1}\right)+\tilde{d}\left(u_{k+1}, u_{k}\right) \\
& \leq \lim _{l \rightarrow \infty} \sum_{j=k}^{l+k} \tilde{d}\left(u_{j}, u_{j+1}\right) \leq \frac{1}{2^{k-1}}
\end{aligned}
$$

Hence, $\left\{v^{k}\right\}_{k \in \mathbb{N}}$ is an increasing $\tilde{d}$-Cauchy sequence that is equivalent to $\left\{u_{k}\right\}_{k \in \mathbb{N}}$. We want to show that $\left\{v^{k}\right\}_{k \in \mathbb{N}}$ increases pointwise a.e. to some $v \in \mathcal{E}^{2}(X, \omega)$.

Using Lemma 9.1 and (52) we have:

$$
0 \leq \frac{1}{n+1} \int_{X}\left(v^{k}-v^{1}\right)\left(\omega+i \partial \bar{\partial} v^{1}\right)^{n} \leq A M\left(v^{k}\right)-A M\left(v^{1}\right) \leq \tilde{d}\left(v^{k}, v^{1}\right) .
$$

Hence, the limit $\tilde{v}=\lim _{k \rightarrow \infty} v^{k}-v^{1} \geq 0$ is finite on a set of capacity non-zero. Indeed, by the monotone convergence theorem $\left(\omega+i \partial \bar{\partial} v^{1}\right)^{n}(\{\tilde{v}=\infty\})=0$, implying $\left(\omega+i \partial \bar{\partial} v^{1}\right)^{n}(\{\tilde{v}<\infty\})=\operatorname{Vol}(X)>0$. As $v^{1} \in \mathcal{E}^{2}(X, \omega) \subset \mathcal{E}(X, \omega)$, by GZ1, Theorem A] it follows that $\operatorname{Cap}(\{\tilde{v}<\infty\})>0$.

Let $A_{l}=\left\{\tilde{v}<l, v^{1}>-l\right\}, l>0$. As $\operatorname{Cap}\left(\left\{v^{1}=-\infty\right\}\right)=0$ and $\left\{\tilde{v}<\infty, v^{1}>\right.$ $-\infty\}=\cup_{l>0} A_{l}$, we conclude that $\operatorname{Cap}\left(A_{l_{0}}\right)>0$ for some $l_{0}>0$ and

$$
-l_{0}<\sup _{A_{l_{0}}} v^{k}<l_{0}+\sup _{A_{l_{0}}} v^{1}, k \in \mathbb{N} .
$$

By [GZ2, Corollary 4.3], this implies that $\left\{v^{k}\right\}_{k \in \mathbb{N}}$ forms an $L^{1}$-relatively compact family in $\operatorname{PSH}(X, \omega)$. It follows that $v_{k}$ increases a.e. to some $v \in \operatorname{PSH}(X, \omega)$, in particular $v \in \mathcal{E}^{2}(X, \omega)$. An application of Proposition 6.11 now yields $\tilde{d}\left(v^{k}, v\right) \rightarrow 0$, which in turn implies $\tilde{d}\left(u_{k}, v\right) \rightarrow 0$.

As promised, we need to argue that decreasing Cauchy sequences in $\left(\mathcal{E}^{2}(X, \omega), \tilde{d}\right)$ have their limit in $\mathcal{E}^{2}(X, \omega)$. Before we do this, we state a lemma of independent interest:

Lemma 9.3. Suppose $u \in \mathcal{H}$ and $u \leq 0$. Then $u / 2 \in \mathcal{H}$ and

$$
d(0, u / 2) \leq d(0, u) / 2 \text {. }
$$


Proof. Suppose $(0,1) \ni t \rightarrow \phi_{t}, \psi_{t} \in \mathcal{H}_{\Delta}$ is the geodesic connecting $0, u$ and $0, u / 2$ respectively. Then $t \rightarrow \phi_{t} / 2$ is a subgeodesic connecting $0, u / 2$. By (15) we have $\tilde{d}(0, u / 2)^{2}=\int_{X} \dot{\psi}_{0}^{2} \omega^{n}$ and $\tilde{d}(0, u)^{2}=\int_{X} \dot{\phi}_{0}^{2} \omega^{n}$. As $\phi_{t} / 2 \leq \psi_{t}, t \in[0,1]$ and both curves are decreasing the desired estimate follows.

Lemma 9.4. Suppose $\left\{u_{k}\right\}_{k \in \mathbb{N}} \subset \mathcal{E}^{2}(X, \omega)$ is a pointwise decreasing $\tilde{d}$-bounded sequence. Then $u=\lim _{k \rightarrow \infty} u_{k} \in \mathcal{E}^{2}(X, \omega)$ and additionally $\tilde{d}\left(u, u_{k}\right) \rightarrow 0$.

Proof. We can suppose that $u_{k}<0$. First we assume that $\left\{u_{k}\right\}_{k \in \mathbb{N}} \subset \mathcal{H}$. By Lemma 9.1 it follows that $u=\lim _{k \rightarrow \infty} u_{k} \in \mathcal{E}^{1}(X, \omega)$. Suppose $v \in \mathcal{E}^{2}(X, \omega)$ and $v<0$. We will prove that

$$
\int_{X} v^{2}(\omega+i \partial \bar{\partial} u)^{n}<+\infty
$$

Suppose $v_{l} \searrow v$ is a decreasing sequence of negative smooth Kähler potentials. As $0>v_{l} \geq 2\left(P\left(v_{l}, u_{k}\right)-u_{k} / 2\right)$ and $\left(\omega+\partial \bar{\partial} u_{k}\right)^{n} \leq 2^{n}\left(\omega+\partial \bar{\partial} u_{k} / 2\right)^{n}$ we can start writing:

$$
\begin{aligned}
\int_{X} v_{l}^{2}\left(\omega+i \partial \bar{\partial} u_{k}\right)^{n} & \leq 2^{n+2} \int_{X}\left(P\left(v_{l}, u_{k}\right)-u_{k} / 2\right)^{2}\left(\omega+i \partial \bar{\partial} u_{k} / 2\right)^{n} \\
& \leq 2^{n+2} \tilde{d}\left(P\left(v_{l}, u_{k}\right), u_{k} / 2\right)^{2} \\
& \leq 2^{n+3}\left(\tilde{d}\left(P\left(v_{l}, u_{k}\right), u_{k}\right)^{2}+\tilde{d}\left(u_{k}, u_{k} / 2\right)^{2}\right) \\
& \leq 2^{n+3}\left(\tilde{d}\left(v_{l}, u_{k}\right)^{2}+\tilde{d}\left(u_{k}, u_{k} / 2\right)^{2}\right) \\
& \leq 2^{n+4}\left(\tilde{d}\left(v_{l}, 0\right)^{2}+2 \tilde{d}\left(u_{k}, 0\right)^{2}+\tilde{d}\left(u_{k} / 2,0\right)^{2}\right)
\end{aligned}
$$

where in the second line we have used Lemma 6.2 and in the forth line we have used the Pythagorean formula (Proposition [8.1). As both $\left\{v_{l}\right\}_{l \in \mathbb{N}}$ and $\left\{u_{k}\right\}_{k \in \mathbb{N}}$ are $\tilde{d}$-bounded sequences, using the previous lemma we conclude that the right hand side above is bounded. Letting $k \rightarrow \infty$ and then $l \rightarrow \infty$ we obtain (53). By [GZ1, Theorem C] and Theorem 2.9 it follows that $u \in \mathcal{E}^{2}(X, \omega)$. The last statement follows from Proposition 6.11

The case when $\left\{u_{k}\right\}_{k \in \mathbb{N}} \subset \mathcal{E}^{2}(X, \omega)$ can be reduced to the above situation using approximation via Proposition 6.11.

\section{References}

[BT] E. Bedford, B. A. Taylor, A new capacity for plurisubharmonic functions, Acta Math. 149 (1982), no. 1-2, 1-40.

[BD] R. Berman, J. P. Demailly, Regularity of plurisubharmonic upper envelopes in big cohomology classes. Perspectives in analysis, geometry and topology, Progr. Math. 296, Birkhäuser/Springer, New York, 2012, 39-66.

[Br] B. Berndtsson, A Brunn-Minkowski type inequality for Fano manifolds and the Bando-Mabuchi uniqueness theorem, arXiv:1103.0923.

[Bl1] Z. Błocki, The complex Monge-Ampère equation in Kähler geometry, CIME Summer School in Pluripotential Theory, Cetraro, July 2011, to appear in Lecture Notes in Mathematics, http://gamma.im.uj.edu.pl/ blocki/publ/ln/cetr.pdf. 
[Bl2] Z. Błocki, On geodesics in the space of Kähler metrics, Proceedings of the "Conference in Geometry" dedicated to Shing-Tung Yau (Warsaw, April 2009), in "Advances in Geometric Analysis", ed. S. Janeczko, J. Li, D. Phong, Advanced Lectures in Mathematics 21, pp. 3-20, International Press, 2012.

[Bl3] Z. Błocki, A gradient estimate in the Calabi-Yau theorem, Math. Ann. 344 (2009), no. 2, 317-327.

[Bl4] Z. Błocki, The complex Monge-Ampre operator in pluripotential theory, lecture notes, http://gamma.im.uj.edu.pl/ blocki/publ/ln/wykl.pdf.

[BK] Z. Błocki, S.Kołodziej, On regularization of plurisubharmonic functions on manifolds, Proceedings of the American Mathematical Society 135 (2007), 2089-2093

[BEGZ] S. Boucksom, P. Eyssidieux, V. Guedj and A. Zeriahi, Monge-Ampère equations in big cohomology classes, Acta Math. 205 (2010), 199-262.

[BH] M. Bridson, A. Haefliger, Metric spaces of non-positive curvature. Grundlehren der Mathematischen Wissenschaften, 319. Springer-Verlag, Berlin, 1999.

[Ca] E. Calabi, The variation of Kähler metrics, Bull. Amer. Math. Soc. 60 (1954), $167-168$

[Cl] S. Calamai, The Calabi metric for the space of Kähler metrics. Math. Ann. 353 (2012), no. 2, 373-402.

[CC] E. Calabi, X. X. Chen, The Space of Kähler Metrics II, J. Differential Geom. 61(2002), no. 2, 173-193.

[C] X. X. Chen, The space of Kähler metrics, J. Differential Geom. 56 (2000), no. 2, $189-234$

[CT] X. X. Chen, G. Tian, Geometry of Kähler metrics and foliations by holomorphic discs, Publ. Math. Inst. Hautes Études Sci. No. 107 (2008), 1-107.

[CR] B. Clarke, Y.A. Rubinstein, Ricci flow and the metric completion of the space of Kähler metrics, American Journal of Mathematics 135 (2013), no. 6, 1477-1505.

[Da1] T. Darvas, Morse theory and geodesics in the space of Kähler metrics, arXiv:1207.4465v3.

[Da2] T. Darvas, Weak Geodesic Rays in the Space of Kähler Metrics and the Class $\mathcal{E}(X, \omega)$, arXiv:1307.6822.

[Da3] T. Darvas, The Mabuchi Geometry of Finite Energy Classes, arXiv:1409.2072.

[DH] T. Darvas, W. He, Geodesic Rays and Kähler-Ricci Trajectories on Fano Manifolds, arXiv:1411.0774.

[DL] T. Darvas, L. Lempert, Weak geodesics in the space of Kähler metrics, Mathematical Research Letters, 19 (2012), no. 5.

[DR] T. Darvas, Y. A. Rubinstein, Kiselman's principle, the Dirichlet problem for the Monge-Ampère equation, and rooftop obstacle problems, arXiv:1405.6548. 
[Di] S. Dinew, Uniqueness in $\mathcal{E}(X, \omega)$. J. Funct. Anal. 256 (2009), no. 7, 2113-2122.

[Do] S. K. Donaldson - Symmetric spaces, Kähler geometry and Hamiltonian dynamics, Amer. Math. Soc. Transl. Ser. 2, vol. 196, Amer. Math. Soc., Providence RI, 1999, 13-33.

[G] V. Guedj, The Metric Completion of the Riemannian Space of Kähler Metrics, preprint.

[GZ1] V. Guedj, A. Zeriahi, The weighted Monge-Ampère energy of quasiplurisubharmonic functions, J. Funct. Anal. 250 (2007), no. 2, 442-482.

[GZ2] V. Guedj, A. Zeriahi, Intrinsic capacities on compact Kähler manifolds. Journal of Geom. An. 15 (2005), no. 4, 607-639.

[GT] D. Gilbarg, N.S. Trudinger, Elliptic partial differential equations of second order. Classics in Mathematics. Springer-Verlag, Berlin, 2001.

[H] W. He, On the space of Kähler potentials, to appear in CPAM, arXiv:1208.1021.

[K] S. Kołodziej, The Monge-Ampère equation on compact Kähler manifolds, Indiana Univ. Math. J. 52 (2003), 667-686.

[LV] L. Lempert, L. Vivas, Geodesics in the space of Kähler metrics. Duke Math. J. 162 (2013), no. 7, 1369-1381.

[M] T. Mabuchi, Some symplectic geometry on compact Kähler manifolds I, Osaka J. Math. 24, 1987, 227-252.

[PS] D.H. Phong, J. Sturm, The Monge-Ampère operator and geodesics in the space of Kähler potentials. Invent. Math. 166 (2006), no. 1, 125-149.

[RWN] J. Ross, D. Witt Nyström, Analytic test configurations and geodesic rays, arXiv:1101.1612.

[RZ] Y. Rubinstein, S. Zelditch, The Cauchy problem for the homogeneous MongeAmpère equation, I. Toeplitz quantization, J. Differential Geom. 90 (2012), no. 2, 303-327.

[Se] S. Semmes, Complex Monge-Ampère and symplectic manifolds, Amer. J. Math. 114 (1992), 495-550.

[SZ] J. Song, S. Zelditch, Bergman metrics and geodesics in the space of Kähler metrics on toric varieties. Anal. PDE 3 (2010), no. 3, 295-358.

[St1] J. Streets, Long time existence of Minimizing Movement solutions of Calabi flow, arXiv:1208.2718.

[St2] J. Streets, The consistency and convergence of K-energy minimizing movements, arXiv:1301.3948.

Department of Mathematics, Purdue University

E-mail address: tdarvas@math.purdue.edu 